\title{
Numerical Solutions for Coupled Trapezoidal Fully Fuzzy Sylvester Matrix Equations
}

\author{
Ahmed AbdelAziz Elsayed (D), ${ }^{1,2}$ Nazihah Ahmad, ${ }^{1}$ and Ghassan Malkawi ${ }^{3}$ \\ ${ }^{1}$ School of Quantitative Sciences, Universiti Utara Malaysia, 06010 Sintok, Kedah, Malaysia \\ ${ }^{2}$ Department of Mathematics, Institute of Applied Technology, Mohamed Bin Zayed City, UAE \\ ${ }^{3}$ Faculty of Engineering, Math and Natural Science Division, Higher Colleges of Technology (HCT), Al Ain, UAE \\ Correspondence should be addressed to Ahmed AbdelAziz Elsayed; ahmed.elsayed1516@gmail.com
}

Received 11 August 2021; Revised 6 October 2021; Accepted 20 November 2021; Published 27 January 2022

Academic Editor: Katsuhiro Honda

Copyright (c) 2022 Ahmed AbdelAziz Elsayed et al. This is an open access article distributed under the Creative Commons Attribution License, which permits unrestricted use, distribution, and reproduction in any medium, provided the original work is properly cited.

\begin{abstract}
Analyzing the stability of many control systems required solving a couple of crisp Sylvester matrix equations (CSMEs) simultaneously. However, there are some situations in which the crisp Sylvester matrix equations are not well equipped to deal with the uncertainty problem during the stability analysis of control systems. This paper constructs analytical and numerical methods for solving a couple of trapezoidal fully fuzzy Sylvester matrix equations (CTrFFSMEs) to overcome the drawbacks of the existing crisp methods. In developing these new methods, fuzzy arithmetic multiplication is applied on the CTrFFSME to transform it into an equivalent system of four CSMEs. Then, the fuzzy solution is obtained analytically by the fuzzy matrix vectorization method and numerically by gradient and least square methods. The analytical method can obtain the exact solution; however, it is limited to small-sized systems while the numerical methods can approximate the solution for large dimensional systems up to $100 \times 100$ with a very small error bound for any initial value. In addition, the proposed methods are applied to other fuzzy systems such as Sylvester and Lyapunov matrix equations. The proposed methods are illustrated by solving numerical examples with different size systems.
\end{abstract}

\section{Introduction}

The Sylvester matrix equation (SME) has massive applications in control theory [1, 2], system theory [3], optima control [4], linear descriptor systems [5], sensitivity analysis [6], perturbation theory [7], system design [8], theory of orbits [9], design and analysis of linear control systems [10], reduction of large-scale dynamical systems [11], restoration of noisy images $[12,13]$, medical imaging data acquisition and model reduction [14], and stochastic control, image processing, and filtering [13]. CSME must be solved simultaneously in many applications, such as analyzing the stability of control systems [15]. Researchers for many years have proposed many analytical and numerical methods for solving CSME with crisp numbers.

Although analytical solutions, which can be computed using Vec-operator and Kronecker product, are important, the computational efforts rapidly increase with the dimensions of the matrices to be solved. For example, it required getting the inverse of $m n \times m n$ matrix for a system of size $m \times n$ which leads to computation complexity. Therefore, this method is limited to systems with small coefficients only. In addition, for some applications such as stability analysis, it is often not necessary to compute analytical solutions; approximate solutions or bounds of solutions are sufficient. Also, if the parameters in system matrices are uncertain, it is not possible to obtain analytical solutions for robust stability results $[16,17]$. Alternative ways exist which transform the matrix equations into forms for which solutions may be readily computed, such as the Jordan canonical form [18] and Hessenberg-Schur form [19]. However, these methods are computationally expensive for large systems. In the field of matrix algebra and system identification, iterative algorithms for large systems have 
received much attention [20]. Starke and Niethammer [21] presented an iterative method for solutions of the SME by using the SOR technique while Jonsson and Kägström proposed recursive block algorithms for solving the coupled Sylvester matrix equations [22]. Kägström derived an approximate solution of the coupled Sylvester equation [23].

Many authors studied the least square solutions of CSME [24-31] while authors in [32] discussed the solvability conditions and general solutions for mixed Sylvester equations. Recently, a relaxed gradient-based algorithm for solving generalized CSME was introduced by [33] in addition to the conjugate gradient least square algorithm [34] and gradient-based approach [35] and the BCR algorithm proposed by [36-38]. However, in many applications, some of the system parameters are represented by fuzzy numbers rather than crisp numbers due to uncertainty problems such as conflicting requirements during the system process and the distraction of any elements and noise. When all parameters of the CSME are in the fuzzy form, then it is called the coupled fully fuzzy Sylvester matrix equation (CFFSME).

Definition 1 . The couple fully fuzzy matrix equation can be written as

$$
\left\{\begin{array}{l}
\widetilde{A} \widetilde{X}+\widetilde{Y} \widetilde{B}=\widetilde{E} \\
\widetilde{C} \widetilde{X}+\widetilde{Y} \widetilde{D}=\widetilde{F}
\end{array}\right.
$$

where $\quad \tilde{A}=\left(\widetilde{a}_{i j}\right)_{m \times m}, \quad \widetilde{X}=\left(\widetilde{x}_{i j}\right)_{m \times n}, \quad \tilde{Y}=\left(\widetilde{y}_{i j}\right)_{m \times n}$, $\widetilde{\widetilde{B}}=\left(\widetilde{b}_{j j}\right)_{n \times n}, \widetilde{C}=\left(\widetilde{c}_{i j}\right)_{m \times m}, \widetilde{D}=\left(\widetilde{d}_{i j}\right)_{n \times n}, \widetilde{E}=\left(\widetilde{e}_{i j}\right)_{m \times n}$, and $\widetilde{F}=\left(\widetilde{f}_{i j}\right)_{m \times n}$.

Equation (1) is of interest in many different applications. However, until now, there are fewer studies for the solution of this equation. In the fuzzy literature, most of the solution methods are proposed for its special cases, such as fully fuzzy Sylvester matrix equations (FFSMEs), fully fuzzy matrix equations (FFMEs), fully fuzzy linear systems (FFLSs), and fuzzy linear system (FLS).

The first approach of solving FLS was accomplished by [39], which proposed a general model for solving a FLS by transferring FLS to a linear system. Allahviranloo et al. [40] proposed a method to obtain symmetric solutions of the FLS based on a 1-cut expansion. They extended the same method in [41] to obtain symmetric solutions of the FFLS. Sufficient conditions needed for getting positive solutions of the FFLS were discussed by Malkawi and his colleagues [42, 43]. Otadi and Mosleh extended the FFLS to FFME in [44]. Several analytical methods have been proposed for solving the triangular fully fuzzy Sylvester matrix equation (TFFSME) [45-47]. However, these methods are restricted only for positive triangular fuzzy numbers and require a long multiplication process and consequently long computational timing. Consequently, researchers limit the sizes of the TFFSME to $n=2$ or 3 . Recently, authors in [48] considered solution of the trapezoidal fully fuzzy Sylvester matrix equation (TrFFSME) by transforming the TrFFSME to a system of crisp linear matrix equations where the positive and negative fuzzy solutions are obtained by applying Kronecker product and Vec-operator method. However, these algorithms are not suitable for TrFFSME with large sizes.

In addition, a few studies have been conducted for solving a pair of fuzzy matrix equations. Sadeghi, Abbasbandy, and Abbasnejad [49] proposed a method for solving a pair of fuzzy matrix equations in the form as follows:

$$
\left\{\begin{array}{l}
A \widetilde{X}+\widetilde{X} B=\widetilde{C}, \\
D \widetilde{X} E=\widetilde{F} .
\end{array}\right.
$$

Moreover, Daud, Ahmad, and Malkawi [50-53] proposed analytical methods for solving FFSME and a pair of fully fuzzy matrix equations (PFFME) in the form as follows:

$$
\left\{\begin{array}{l}
\widetilde{A} \widetilde{X}+\widetilde{X} \widetilde{B}=\widetilde{C}, \\
\widetilde{D} \widetilde{X} \widetilde{E}=\widetilde{F} .
\end{array}\right.
$$

In that study, a direct method was proposed to solve the PFFME by applying the Kronecker product and Vec-operator. However, both methods required a long multiplication process and were consequently limited to small-sized systems. In general, the existing methods proposed for solving PFFME, TFFSME, and TrFFSME are based on Kronecker product and Vec-operator and therefore limited to small systems $(2 \times 2)$ or $(3 \times 3)$. Only a few researchers considered fuzzy systems with sizes $10 \times 10$ [43]. Fuzzy systems with sizes greater than $10 \times 10$ are not investigated till now. In addition, the CFFSME is not investigated in the fuzzy literature.

To deal with this shortcoming, in this paper, three different methods are proposed for solving CFFSME with trapezoidal fuzzy numbers (CTrFFSME) and its special cases. The fuzzy solution to the CTrFFSME is obtained analytically by the fuzzy matrix vectorization method and numerically by gradient and least square methods. The fuzzy matrix vectorization method can obtain the exact solution; however, it is restricted to small systems. Therefore, it is important to develop mathematical models and numerical procedures that solve the CFFSME and special cases with big sizes while the numerical methods can obtain the solution for large dimensional systems up to $100 \times 100$ with a very small error bound compared with the existing numerical approaches, which were applied up to $10 \times 10$ fuzzy systems [54-63]. Moreover, the proposed methods can also be applied to other fuzzy systems such as Sylvester and Lyapunov matrix equations with triangular fuzzy numbers (TFNs) and trapezoidal (TrFNs) fuzzy numbers.

To illustrate the effectiveness of the proposed methods for solving the CTrFFSME in equation (1), we consider various sizes of fuzzy systems, namely, small $2 \times 2$ and large $100 \times 100$. In addition, we compare the performance of the proposed methods by calculating the number of iterations $(k)$, convergence factor $(\alpha)$, error $\delta^{l}(k)$, error bound $(\varepsilon)$, convergence rate, CPU time, real-time, and memory usage. In addition to the graphical representation of the relative error $\delta^{l}(k)$ when the number of iterations $(k)$ increases.

This paper is organized as follows. Section 2 introduces preliminary arithmetic operations of trapezoidal fuzzy numbers. In Section 3, three proposed methods for solving 
CTrFFSME are developed along with a presentation of its algorithms. In Section 4, numerical examples are presented to illustrate the proposed methods. Section 5 is dedicated to the conclusion.

\section{Preliminaries}

The following are the basic definitions and results related to TrFNs in fuzzy theory [64-66] and matrix theory [67-69].

Definition 2. Let $X$ be a universal set. Then, the fuzzy subset $\widetilde{A}$ of $X$ is defined by its membership function $\mu_{A}: X \longrightarrow[0,1]$ which assigns to each element $x \in X$ a real number $\mu_{\tilde{A}}(x)$ in the interval $[0,1]$, where the function value of $\mu_{\tilde{A}}(x)$ represents the grade of membership of $x$ in $\widetilde{A}$. A fuzzy set $\widetilde{A}$ is written as $\widetilde{A}=\left\{\left(x, \mu_{\tilde{A}}(x)\right)\right.$, $\left.x \in X, \mu_{\tilde{A}}(x) \in[0,1]\right\}$.

Definition 3. A fuzzy set $\widetilde{A}$, defined on the universal set of real number $R$, is said to be a fuzzy number if its membership function has the following characteristics:

(i) $\widetilde{A}$ is convex, i.e.,

$$
\begin{aligned}
\mu_{A}\left(\lambda x_{1}+(1-\lambda)\right) x_{2} & \geq \min \left(\mu_{\tilde{A}}(x), \mu_{\tilde{A}}(x)\right) \\
& \forall x_{1}, x_{2} \in R, \forall \lambda \in[0,1] .
\end{aligned}
$$

(ii) $\widetilde{A}$ is normal, i.e.,

$$
\exists x_{0} \in R \text { such that } \mu_{\tilde{A}}\left(x_{0}\right)=1 .
$$

(iii) $\mu_{A} \sim$ is piecewise continuous.

Definition 4. A fuzzy number $\widetilde{A}=\left(a_{1}, a_{2}, a_{3}, a_{4}\right)$ is a $\operatorname{TrFN}$ in the general form if its membership function is as follows:

$$
\mu_{A}(x)= \begin{cases}0, & x<a_{1}, \\ \frac{x-a_{1}}{a_{2}-a_{1}}, & a_{1} \leq x \leq a_{2}, \\ 1, & a_{2} \leq x \leq a_{3}, \\ \frac{a_{4}-x}{a_{4}-a_{3}}, & a_{3} \leq x \leq a_{4}, \\ 0, & x>a_{4} .\end{cases}
$$

In Figure 1, the $\operatorname{TrFN}$ in general form is presented.

Definition 5. The sign of the $\operatorname{TrFN} \widetilde{A}=\left(a_{1}, a_{2}, a_{3}, a_{4}\right)$ can be classified as follows:

$\widetilde{A}$ is positive (negative) iff $a_{1} \geq 0,\left(a_{4} \leq 0\right)$

$\widetilde{A}$ is zero iff $\left(a_{1}, a_{2}, a_{3}\right.$ and $\left.a_{4}=0\right)$

$\widetilde{A}$ is near zero iff $a_{1} \leq 0 \leq a_{4}$

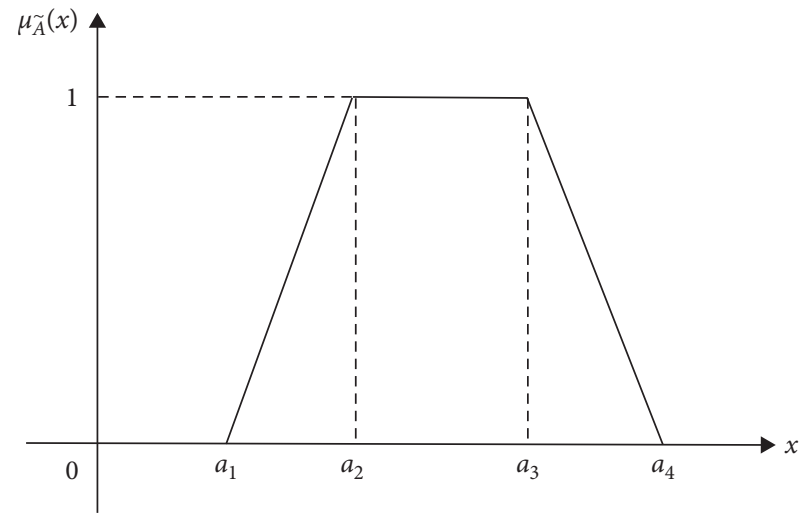

Figure 1: Representation of $\operatorname{TrFN}$ in a general form.

Definition 6. Operations of TrFNs.

The arithmetic operations of TrFNs are presented as follows: let $\widetilde{A}=\left(a_{1}, a_{2}, a_{3}, a_{4}\right)$ and $\widetilde{B}=\left(b_{1}, b_{2}, b_{3}, b_{4}\right)$ be two TrFNs, then

(i) Addition:

$$
\begin{aligned}
\widetilde{A}+\widetilde{B} & =\left(a_{1}, a_{2}, a_{3}, a_{4}\right)+\left(b_{1}, b_{2}, b_{3}, b_{4}\right) \\
& =\left(a_{1}+b_{1}, a_{2}+b_{2}, a_{3}+b_{3}, a_{4}+b_{4}\right) .
\end{aligned}
$$

(ii) Subtraction:

$$
\begin{aligned}
\widetilde{A}-\widetilde{B} & =\left(a_{1}, a_{2}, a_{3}, a_{4}\right)-\left(b_{1}, b_{2}, b_{3}, b_{4}\right) \\
& =\left(a_{1}-b_{4}, a_{2}-b_{3}, a_{3}-b_{2}, a_{4}-b_{1}\right) .
\end{aligned}
$$

(iii) Symmetric image:

$$
-\widetilde{A}=\left(-a_{4},-a_{3},-a_{2},-a_{1}\right) .
$$

(iv) Scalar multiplication: let $\lambda \in \mathbb{R}$, then

$$
\lambda \otimes\left(a_{1}, a_{2}, a_{3}, a_{4}\right)= \begin{cases}\left(\lambda a_{1}, \lambda a_{2}, \lambda a_{3}, \lambda a_{4}\right), & \lambda \geq 0, \\ \left(\lambda a_{4}, \lambda a_{3}, \lambda a_{2}, \lambda a_{1}\right), & \lambda<0 .\end{cases}
$$

(v) Multiplication: the multiplication between fuzzy numbers is neither commutative nor associative. Thus, TrFNs multiplication operations can be classified as follows:

Case I. If $\widetilde{A}=\left(a_{1}, a_{2}, a_{3}, a_{4}\right)$ and $\widetilde{B}=\left(b_{1}, b_{2}, b_{3}, b_{4}\right)$ be two arbitrary TrFNs, then

$$
\widetilde{A} \widetilde{B}=(a, h, m, d),
$$

where

$$
\begin{aligned}
a & =\min \left(a_{1} b_{1}, a_{1} b_{4}, a_{4} b_{1}, a_{4} b_{4}\right), \\
h & =\min \left(a_{2} b_{2}, a_{2} b_{3}, a_{3} b_{2}, a_{3} b_{3}\right), \\
m & =\max \left(a_{2} b_{2}, a_{2} b_{3}, a_{3} b_{2}, a_{3} b_{3}\right), \\
d & =\max \left(a_{1} b_{1}, a_{1} b_{4}, a_{4} b_{1}, a_{4} b_{4}\right) .
\end{aligned}
$$

Case II. If $\widetilde{A}, \widetilde{B}>0$, then

$$
\widetilde{A} \widetilde{B}=\left(a_{1} b_{1}, a_{2} b_{2}, a_{3} b_{3}, a_{4} b_{4}\right) .
$$


Case III. If $\widetilde{A}, \widetilde{B}<0$, then

$$
\widetilde{A} \widetilde{B}=\left(a_{4} b_{4}, a_{3} b_{3}, a_{2} b_{2}, a_{1} b_{1}\right) .
$$

Case IV. If $\widetilde{A}>0$ and $\widetilde{B}<0$, then

$$
\widetilde{A} \widetilde{B}=\left(a_{4} b_{1}, a_{3} b_{2}, a_{2} b_{3}, a_{1} b_{4}\right) .
$$

Case $V$. If $\widetilde{A}<0$ and $\widetilde{B}>0$, then

$$
\widetilde{A} \widetilde{B}=\left(a_{1} b_{4}, a_{2} b_{3}, a_{3} b_{2}, a_{4} b_{1}\right) \text {. }
$$

(vi) Equality: the fuzzy numbers $\widetilde{A}=\left(a_{1}, a_{2}, a_{3}, a_{4}\right)$ and $\widetilde{B}=\left(b_{1}, b_{2}, b_{3}, b_{4}\right)$ are equal iff

$$
\begin{aligned}
& a_{1}=b_{1}, \\
& a_{2}=b_{2}, \\
& a_{3}=b_{3}, \\
& a_{4}=b_{4} .
\end{aligned}
$$

Definition 7. A matrix $\widetilde{A}=\left(\widetilde{a}_{i j}\right)_{m \times n}$ is called a trapezoidal fuzzy matrix if each element of $\widetilde{A}$ is a $\operatorname{TrFN}$.

Definition 8. A fuzzy matrix $\widetilde{A}$ will be as follows:

(i) Positive (negative) and denoted by $\widetilde{A}>0,(\widetilde{A}<0)$ if each element of $\widetilde{A}$ is positive (negative) $\operatorname{TrFN}$

(ii) Nonnegative (nonpositive) and denoted by $\widetilde{A} \geq 0$, $(\widetilde{A} \leq 0)$ if each element of $\widetilde{A}$ is nonnegative (nonpositive) TrFNs

(iii) Arbitrary if at least one element of $\widetilde{A}$ is near zero TrFNs

In Remark 1, the positive trapezoidal fuzzy matrix is written as four separated crisp matrices.

Remark 1. The positive trapezoidal fuzzy matrices $\widetilde{A}=\left(\tilde{a}_{i j}\right)_{m \times m}$ can be written as four separated crisp matrices as follows:

$$
\widetilde{A}=\left(\widetilde{a}_{i j}\right)_{m \times m}=\left(A^{(1)}, A^{(2)}, A^{(3)}, A^{(4)}\right),
$$

where $A^{(1)}, A^{(2)}, A^{(3)}$, and $A^{(4)}$ are four crisp matrices sized $m \times m$.

In Remark 2, the multiplication of positive trapezoidal fuzzy matrices is introduced.

Remark 2. The product of the two positive trapezoidal fuzzy matrices $\quad \widetilde{A}=\left(\widetilde{a}_{i j}\right)_{m \times m}=\left(A^{(1)}, A^{(2)}, A^{(3)}, A^{(4)}\right)$, $\forall 1 \leq i, j \leq m$, and $\tilde{X}=\left(\tilde{x}_{p q}\right)_{m \times n}=\left(X^{(1)}, X^{(2)}, X^{(3)}, X^{(4)}\right)$, $\forall 1 \leq p \leq m, 1 \leq q \leq n$, can be represented as follows:

$$
\tilde{A} \widetilde{X}=\sum_{k=1}^{n} \tilde{a}_{i k} \tilde{x}_{k q},
$$

where $\tilde{a}_{i k} \tilde{x}_{k q}$ represent the multiplication of the fuzzy $k-$ th number of $i$ - row of matrix $\widetilde{A}$ with $q$ - column of matrix $\widetilde{X}$.
In addition, this product is equivalent to the product of the following crisp matrices:

$$
\tilde{A} \widetilde{X}=\left(A^{(1)} X^{(1)}, A^{(2)} X^{(2)}, A^{(3)} X^{(3)}, A^{(4)} X^{(4)}\right) \text {. }
$$

Definition 9. The Vec-operator generates a column vector from a matrix $A$ by stacking the column vectors of $A=$

$$
\begin{aligned}
& \left(\begin{array}{ccc}
a_{11} & \cdots & a_{1 n} \\
\vdots & \ddots & \vdots \\
a_{n 1} & \cdots & a_{n n}
\end{array}\right) \text { as } \operatorname{Vec}(A)=\left(\begin{array}{c}
a_{11} \\
a_{21} \\
\vdots \\
a_{n n}
\end{array}\right) \text {. In addition, if } \\
& A=\operatorname{Vec}^{-1}\left(\begin{array}{c}
a_{11} \\
a_{21} \\
\vdots \\
a_{n n}
\end{array}\right) \text {, then } A=\left(\begin{array}{ccc}
a_{11} & \cdots & a_{1 n} \\
\vdots & \ddots & \vdots \\
a_{n 1} & \cdots & a_{n n}
\end{array}\right) .
\end{aligned}
$$

Theorem 1 (see [70]). If the crisp linear matrix equation $A X=E$ has a unique solution $X$, then the gradient iterative solution $X(k)$ given by $X(k)=X(k-1)+$ $\alpha \cdot(A)^{T}\left(E_{\wedge}-A X(k-1)\right) \quad$ converges ${ }_{\wedge}$ to $X$ or $\lim _{k \rightarrow \infty}(X(k))=X$ for any initial value $X(0)$.

Theorem 2 (see [71]). If the crisp linear matrix equation $A X=E$ has a unique solution $X$, then the gradient iterative solution $X(k)$ given $\wedge$ by $X(k)=X(k-1)+$ $\alpha \cdot\left((A)^{T} \cdot A\right)^{-1}(A)^{T}(E-A X(k-1))$ converges to $X$ or $\lim _{k \rightarrow \infty}(X(k))=X$ for any initial value $X(0)$.

In Section 3, the solution to the CTrFFSME in equation (1) is discussed.

\section{The Solution of Coupled Trapezoidal Fully Fuzzy Sylvester Matrix Equation}

In this section, the solution to the positive CTrFFSME is considered. To get the solution, the positive CTrFFSME is converted to an equivalent system of CSME, and then the solution to this system of CSME is obtained by three different methods. In Section 3.1, the positive CTrFFSME in equation (1) is converted to an equivalent system CSME based on the arithmetic multiplication operation in Definition 6 .

3.1. Systems of CSME. In this section, the positive CTrFFSME in equation (1) is converted to four systems of CSME. The next theorem shows that the CTrFFSME can be written as four systems of CSME.

Theorem 3. Fundamental theorem of the coupled trapezoidal fully fuzzy Sylvester matrix equation.

In the CTrFFSME in equation (1), if $\widetilde{A}=\left(\widetilde{a}_{i j}\right)_{m \times m}=\left(A^{(1)}\right.$, $\left.A^{(2)}, A^{(3)}, A^{(4)}\right)>0$ and $\widetilde{C}=\left(\widetilde{c}_{i j}\right)_{m \times m}=\left(C^{(1)}, C^{(2)}, C^{(3)}, C^{(4)}\right)$ $>0, \forall 1 \leq i, j \leq m, \widetilde{B}=\left(\widetilde{b}_{i j}\right)_{n \times n}=\left(B^{(1)}, B^{(2)}, B^{(3)}, B^{(4)}\right)>0$ and $\widetilde{D}=\left(\tilde{d}_{i j}\right)_{n \times n}=\left(D^{(1)}, D^{(2)}, D^{(3)}, D^{(4)}\right)>0, \forall 1 \leq i, j \leq n$, and $\widetilde{X}=$ $\left(\tilde{x}_{i j}\right)_{m \times n}=\left(X^{(1)}, X^{(2)}, \quad X^{(3)}, X^{(4)}\right)>0, \quad \tilde{Y}=\left(\tilde{y}_{i j}\right)_{m \times n}=\left(Y^{(1)}\right.$, $\left.Y^{(2)}, Y^{(3)}, Y^{(4)}\right)>0, \quad \widetilde{E}=\left(\widetilde{e}_{i j}\right)_{m \times n}=\left(E^{(1)}, E^{(2)}, E^{(3)}, E^{(4)}\right)>0$, 
and $\quad \widetilde{F}=\left(\tilde{f}_{i j}\right)_{m \times n}=\quad\left(F^{(1)}, F^{(2)}, F^{(3)}, F^{(4)}\right)>0$, $\forall 1 \leq i \leq m, 1 \leq j \leq n$, then the positive CTrFFSME is equivalent to the following systems of CSME:

$$
\begin{cases}\sum_{k=1}^{n} \widetilde{a}_{i k} \widetilde{x}_{k j}+\sum_{k=1}^{n} \widetilde{y}_{i k} \widetilde{b}_{k j}=\widetilde{e}_{i j}, \quad \forall 1 \leq i \leq m, 1 \leq j \leq n, \\ \sum_{k=1}^{n} \widetilde{c}_{i k} \widetilde{x}_{k j}+\sum_{k=1}^{n} \widetilde{y}_{i k} \widetilde{d}_{k j}=\widetilde{f}_{i j}, \quad \forall 1 \leq i \leq m, 1 \leq j \leq n .\end{cases}
$$

Proof. Since $\widetilde{A}, \widetilde{B}, \widetilde{C}, \widetilde{D}, \widetilde{E}, \widetilde{F}, \widetilde{X}$, and $\widetilde{Y}$ in equation (1) are positive trapezoidal fully fuzzy matrices, respectively, equation (12) in Definition 6 can be used to find $\widetilde{A} \widetilde{X}, \widetilde{Y} \widetilde{B}$, $\widetilde{C} \widetilde{X}$, and $\widetilde{Y} \widetilde{D}$ in equation (1) as follows:

$$
\begin{aligned}
& \widetilde{A} \widetilde{X}=\sum_{k=1}^{n} \widetilde{a}_{i k} \widetilde{x}_{k j}=\left(A^{(1)} X^{(1)}, A^{(2)} X^{(2)}, A^{(3)} X^{(3)}, A^{(4)} X^{(4)}\right), \\
& \widetilde{Y} \widetilde{B}=\sum_{k=1}^{n} \widetilde{y}_{i k} \widetilde{b}_{k j}=\left(Y^{(1)} B^{(1)}, Y^{(2)} B^{(2)}, Y^{(3)} B^{(3)}, Y^{(4)} B^{(4)}\right), \\
& \widetilde{C} \widetilde{X}=\sum_{k=1}^{n} \widetilde{c}_{i k} \tilde{x}_{k j}=\left(C^{(1)} X^{(1)}, C^{(2)} X^{(2)}, C^{(3)} X^{(3)}, C^{(4)} X^{(4)}\right), \\
& \widetilde{Y} \widetilde{D}=\sum_{k=1}^{n} \tilde{y}_{i k} \tilde{d}_{k j}=\left(Y^{(1)} D^{(1)}, Y^{(2)} D^{(2)}, Y^{(3)} D^{(3)}, Y^{(4)} D^{(4)}\right),
\end{aligned}
$$

s.t all $i=1, \ldots, m$ and $j=1, \ldots, n$. Combining $\tilde{A} \widetilde{X}$ and $\tilde{Y} \widetilde{B}$, $\widetilde{C} \widetilde{X}$ and $\widetilde{Y} \widetilde{D}$, we get

$$
\begin{cases}\sum_{k=1}^{n} \tilde{a}_{i k} \tilde{x}_{k j}+\sum_{k=1}^{n} \tilde{y}_{i k} \widetilde{b}_{k j}, \quad \forall 1 \leq i \leq m, 1 \leq j \leq n, \\ \sum_{k=1}^{n} \widetilde{d}_{i k} \tilde{x}_{k j}+\sum_{k=1}^{n} \tilde{y}_{i k} \widetilde{e}_{k j}, \quad \forall 1 \leq i \leq m, 1 \leq j \leq n .\end{cases}
$$

By Definition 1, the positive CTrFFSME is equivalent to the following systems of CSME:

$$
\begin{cases}\sum_{k=1}^{n} \tilde{a}_{i k} \tilde{x}_{k j}+\sum_{k=1}^{n} \tilde{y}_{i k} \widetilde{b}_{k j}=\widetilde{e}_{i j}, & \forall 1 \leq i \leq m, 1 \leq j \leq n, \\ \sum_{k=1}^{n} \tilde{c}_{i k} \tilde{x}_{k j}+\sum_{k=1}^{n} \tilde{y}_{i k} \tilde{d}_{k j}=\tilde{f}_{i j}, & \forall 1 \leq i \leq m, 1 \leq j \leq n .\end{cases}
$$

Remark 3. The equivalent systems of CSME in equation (20) to the CTrFFSME in equation (1) can also be written as follows:

$$
\left\{\begin{array}{l}
\left\{\begin{array}{l}
A^{(1)} X^{(1)}+Y^{(1)} B^{(1)}=E^{(1)}, \\
C^{(1)} X^{(1)}+Y^{(1)} D^{(1)}=F^{(1)},
\end{array}\right. \\
\left\{\begin{array}{l}
A^{(2)} X^{(2)}+Y^{(2)} B^{(2)}=E^{(2)}, \\
C^{(2)} X^{(2)}+Y^{(2)} D^{(2)}=F^{(2)}
\end{array}\right. \\
\left\{\begin{array}{l}
A^{(3)} X^{(3)}+Y^{(3)} B^{(3)}=E^{(3)}, \\
C^{(3)} X^{(3)}+Y^{(3)} D^{(3)}=F^{(3)},
\end{array}\right. \\
\left\{\begin{array}{l}
A^{(4)} X^{(4)}+Y^{(4)} B^{(4)}=E^{(4)}, \\
C^{(4)} X^{(4)}+Y^{(4)} D^{(4)}=F^{(4)}
\end{array}\right.
\end{array}\right.
$$

To solve the CTrFFSME in equation (1), we consider the corresponding systems of CSME in equation (24).

Remark 4. The nature of the solutions of the CTrFFSME in equation (1) depends upon the nature of the solutions of the system of CSME in equation (24), which may be unique, trivial, or infinitely many solutions [72]; that is, the CTrFFSME may yield no solution, unique solution, or infinitely many solutions.

Since the systems of CSME obtained in equation (24) are similar, in Remark 5, the systems of CSME are represented in a more general form.

Remark 5. Based on equation (24), the CTrFFSME in equation (1) can be written as follows: for $1 \leq l \leq 4$, we have

$$
\left\{\begin{array}{l}
A^{(l)} X^{(l)}+Y^{(l)} B^{(l)}=E^{(l)}, \\
C^{(l)} X^{(l)}+Y^{(l)} D^{(l)}=F^{(l)} .
\end{array}\right.
$$

Based on Theorem 3, the CTrFFSME in equation (1) is transferred to an equivalent linear system of CSME in crisp form, which can be solved analytically and numerically by many classical methods in linear algebra like the matrix inversion method or Gaussian method. The main advantage of the analytical methods is that the exact fuzzy solution to the CTrFFSME in equation (1) can be obtained.

However, since most of the analytical methods are based on Vec-operator and Kronecker products, the system's size in equation (1) is limited to small sizes $(n<10)$. For CTrFFSME with large dimensions $(n \geq 10)$, iterative algorithms to find an approximated solution are more practical [20]. Therefore, in the following section, three different methods are proposed for solving the CTrFFSME in equation (1). The first method aims to find the exact fuzzy solution by extending the concept of matrix vectorization and the Kronecker product. In addition, two iterative methods 
are applied to approximate the fuzzy solution of the CTrFFSME with large dimensions. In Definition 10, the positive trapezoidal fuzzy solution is defined.

Definition 10. Trapezoidal positive fuzzy solution matrix in a general form.

Let $\widetilde{X}=\left(\widetilde{x}_{i j}\right)_{m \times n}=\left(X^{(1)}, X^{(2)}, X^{(3)}, X^{(4)}\right)$ be a trapezoidal fuzzy matrix. If $\widetilde{X}=\left(X^{(1)}, X^{(2)}, X^{(3)}, X^{(4)}\right)$ is an exact solution of equation (20) such that $0<X^{(1)} \leq$ $X^{(2)} \leq X^{(3)} \leq X^{(4)}, \quad \forall 1 \leq i, j \leq m, n, \quad$ then $\quad \tilde{X}=\left(X^{(1)}\right.$, $\left.X^{(2)}, X^{(3)}, X^{(4)}\right)$ is called a positive fuzzy solution of equation (1).

3.2. Proposed Analytical Method for Solving CTrFFSME. In the following method, the analytical solution for the CTrFFSME in equation (1) is obtained by extending the method of the fuzzy matrix vectorization method (FMVM) proposed by [48].

3.2.1. Fuzzy Matrix Vectorization Method (FMVM) for Solving CTrFFSME. In this method, the CTrFFSME in equation (1) is solved analytically using Vec-Operator and Kronecker product. The following steps summarize the methods:

Step 1. Decompose $\widetilde{A}, \widetilde{B}, \widetilde{C}, \widetilde{D}, \widetilde{E}, \widetilde{F}, \widetilde{X}$, and $\widetilde{Y}$ into $A^{(l)}$, $B^{(l)}, C^{(l)}, D^{(l)}, E^{(l)}, F^{(l)}, X^{(l)}$, and $Y^{(l)}$ where $l=1,2,3,4$, respectively, and convert the CTrFFSME in equation (1) to the system of linear matrix equations in equation (24) using Theorem 3.

Step 2. Applying Vec-operator and Kronecker product on equation (24) gives the following:

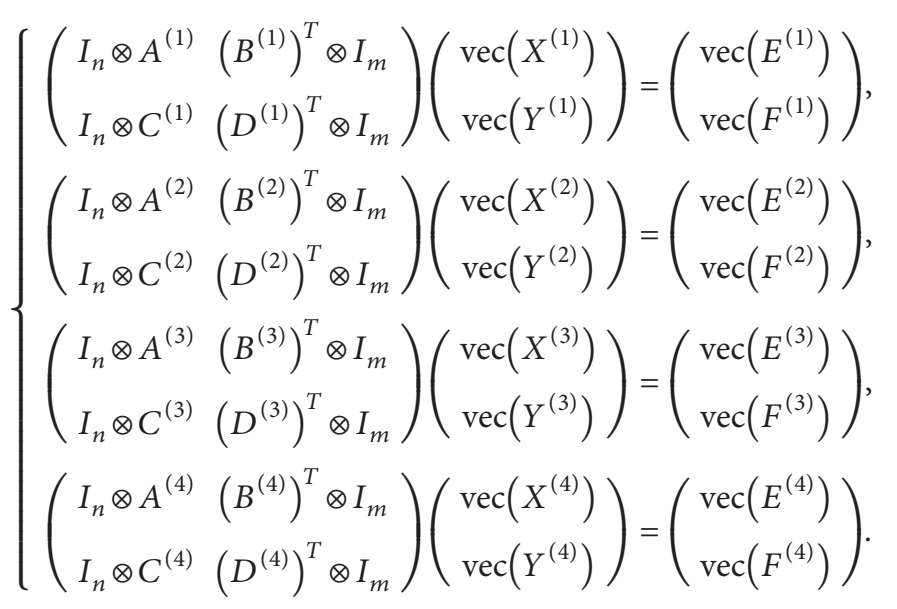

Step 3. By equation (25), the systems of coupled linear matrix equations in equation (27) can be combined and written as follows:

$$
P Q=U,
$$

where 


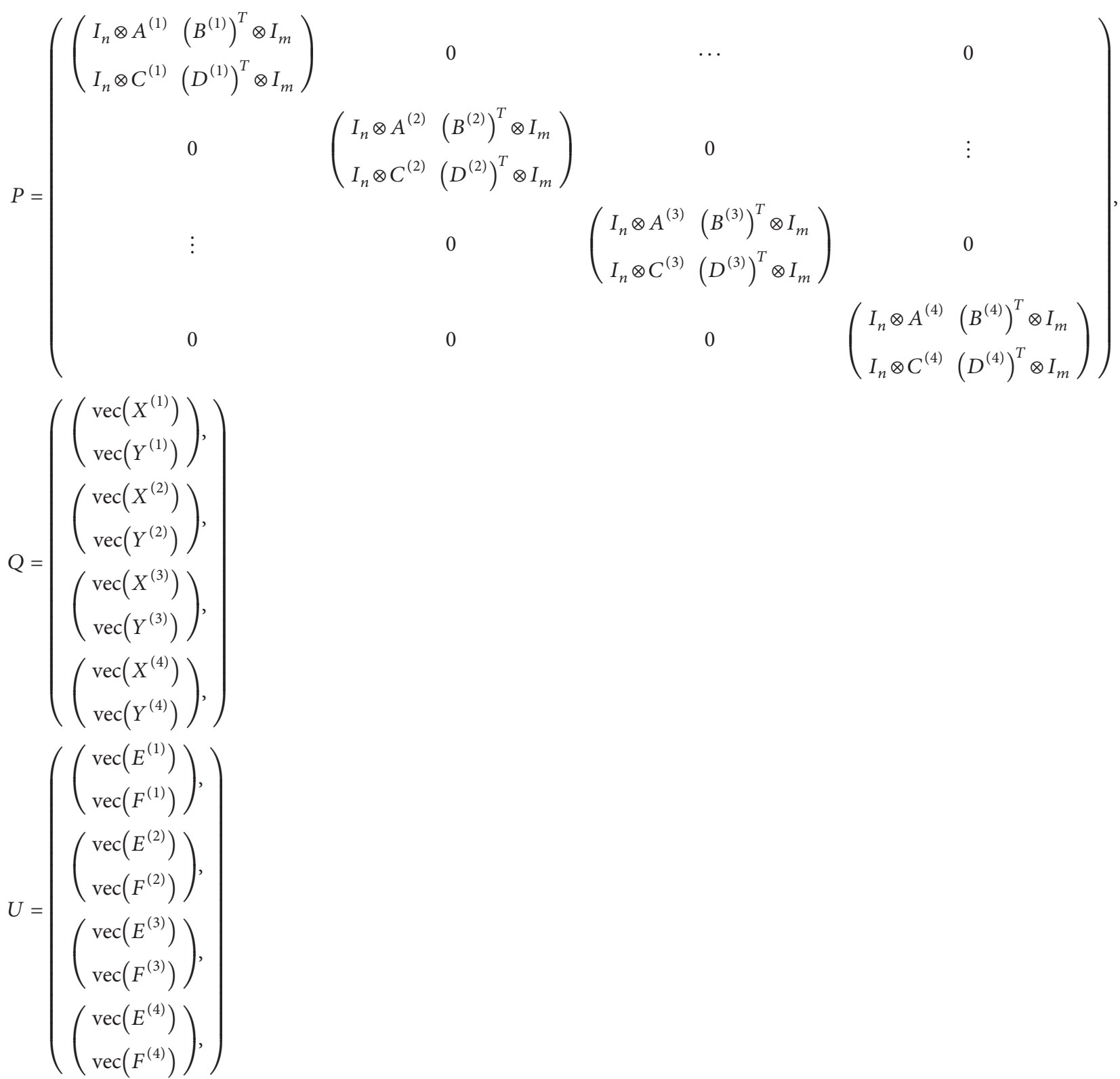

where the crisp matrix $P$ is $8 m n \times 8 m n$, and the crisp vectors $Q$ and $U$ are $8 m n \times 1$.

Step 4. Multiplying the system of equation in equation (27) by matrix multiplicative inverse gives

$$
Q=P^{-1} U \text {. }
$$

Step 5: By Definitions 9 and 10, the obtained solution in equation (29) can be written as follows:

$$
\left\{\begin{array}{c}
\tilde{X}=\left(\begin{array}{ccc}
\left(x_{11}^{(1)}, x_{11}^{(2)}, x_{11}^{(3)}, x_{11}^{(4)}\right) & \cdots & \left(x_{1 n}^{(1)}, x_{1 n}^{(2)}, x_{1 n}^{(3)}, x_{1 n}^{(4)}\right) \\
\vdots & \ddots & \vdots \\
x_{m 1}^{(1)}, x_{m 1}^{(2)}, x_{m 1}^{(3)}, x_{m 1}^{(4)} & \cdots & x_{m n}^{(1)}, x_{m n}^{(2)}, x_{m n}^{(3)}, x_{m n}^{(4)}
\end{array}\right), \\
\widetilde{Y}=\left(\begin{array}{ccc}
\left(y_{11}^{(1)}, y_{11}^{(2)}, y_{11}^{(3)}, y_{11}^{(4)}\right) & \cdots & \left(y_{1 n}^{(1)}, y_{1 n}^{(2)}, y_{1 n}^{(3)}, y_{1 n}^{(4)}\right) \\
\vdots & \ddots & \vdots \\
y_{m 1}^{(1)}, y_{m 1}^{(2)}, y_{m 1}^{(3)}, y_{m 1}^{(4)} & \cdots & y_{m n}^{(1)}, y_{m n}^{(2)}, y_{m n}^{(3)}, y_{m n}^{(4)}
\end{array}\right) .
\end{array}\right.
$$


In the following remark, the system of equations in equation (26) is written in a general form.

Remark 6. Based on equations (25) and (27), the CTrFFSME in equation (1) is equivalent to the following system:for $1 \leq l \leq 4$, we have

$$
\left(\begin{array}{cc}
I_{n} \otimes A^{(l)} & \left(B^{(l)}\right)^{T} \otimes I_{m} \\
I_{n} \otimes C^{(l)} & \left(D^{(l)}\right)^{T} \otimes I_{m}
\end{array}\right)\left(\begin{array}{c}
\operatorname{vec}\left(X^{(l)}\right) \\
\operatorname{vec}\left(Y^{(l)}\right)
\end{array}\right)=\left(\begin{array}{c}
\operatorname{vec}\left(E^{(l)}\right) \\
\operatorname{vec}\left(F^{(l)}\right)
\end{array}\right) .
$$

The sufficient conditions to have a unique fuzzy solution to the CTrFFSME are discussed in Corollary 1.

Corollary 1. For $1 \leq l \leq 4$, the CTrFFSME in equation (1) has a unique solution if and only if the matrix $\left(\begin{array}{cc}I_{n} \otimes A^{(l)} & \left(B^{(l)}\right)^{T} \otimes I_{m} \\ I_{n} \otimes C^{(l)} & \left(D^{(l)}\right)^{T} \otimes I_{m}\end{array}\right)$ is nonsingular. Then, this solution is obtained by

$$
\left(\begin{array}{c}
\operatorname{vec}\left(X^{(l)}\right) \\
\operatorname{vec}\left(Y^{(l)}\right)
\end{array}\right)=\left(\begin{array}{cc}
I_{n} \otimes A^{(l)} & \left(B^{(l)}\right)^{T} \otimes I_{m} \\
I_{n} \otimes C^{(l)} & \left(D^{(l)}\right)^{T} \otimes I_{m}
\end{array}\right)^{-1}\left(\begin{array}{c}
\operatorname{vec}\left(E^{(l)}\right) \\
\operatorname{vec}\left(F^{(l)}\right)
\end{array}\right),
$$

and can be written as

$$
\left\{\begin{array}{c}
\widetilde{X}=\left(\begin{array}{ccc}
\left(x_{11}^{(1)}, x_{11}^{(2)}, x_{11}^{(3)}, x_{11}^{(4)}\right) & \cdots & \left(x_{1 n}^{(1)}, x_{1 n}^{(2)}, x_{1 n}^{(3)}, x_{1 n}^{(4)}\right) \\
\vdots & \ddots & \vdots \\
x_{m 1}^{(1)}, x_{m 1}^{(2)}, x_{m 1}^{(3)}, x_{m 1}^{(4)} & \cdots & x_{m n}^{(1)}, x_{m n}^{(2)}, x_{m n}^{(3)}, x_{m n}^{(4)}
\end{array}\right), \\
\widetilde{Y}=\left(\begin{array}{ccc}
\left(y_{11}^{(1)}, y_{11}^{(2)}, y_{11}^{(3)}, y_{11}^{(4)}\right) & \cdots & \left(y_{1 n}^{(1)}, y_{1 n}^{(2)}, y_{1 n}^{(3)}, y_{1 n}^{(4)}\right) \\
\vdots & \ddots & \vdots \\
y_{m 1}^{(1)}, y_{m 1}^{(2)}, y_{m 1}^{(3)}, y_{m 1}^{(4)} & \cdots & y_{m n}^{(1)}, y_{m n}^{(2)}, y_{m n}^{(3)}, y_{m n}^{(4)}
\end{array}\right) .
\end{array}\right.
$$

3.2.2. Feasibility of the Positive Fuzzy Solution to the CTrFFSME. The obtained positive fuzzy solution in equation (30) to the CTrFFSME in equation (1) is feasible (strong fuzzy solution) if the following conditions are satisfied: for $1 \leq l \leq 4$,

$$
\begin{aligned}
& \text { (i) } X^{(l)}>0, \forall\{1 \leq i, j \leq m, n\} \\
& \text { (ii) } Y^{(l)}>0, \forall\{1 \leq i, j \leq m, n\} \\
& \text { (iii) } X^{(1)} \leq X^{(2)} \leq X^{(3)} \leq X^{(4)}, \forall\{1 \leq i, j \leq m, n\} \\
& \text { (iv) } Y^{(1)} \leq Y^{(2)} \leq Y^{(3)} \leq Y^{(4)}, \forall\{1 \leq i, j \leq m, n\}
\end{aligned}
$$

Remark 7. If the solution fails to satisfy the feasibility conditions, it is infeasible (weak fuzzy solution).

The algorithm of the FMVM for solving the CTrFFMSE in equation (1) is given in the following five steps.
$\left\{\begin{array}{c}\tilde{X}=\left(\begin{array}{ccc}\left(x_{11}^{(1)}, x_{11}^{(2)}, x_{11}^{(3)}, x_{11}^{(4)}\right) & \cdots & \left(x_{1 n}^{(1)}, x_{1 n}^{(2)}, x_{1 n}^{(3)}, x_{1 n}^{(4)}\right) \\ \vdots & \ddots & \vdots \\ x_{m 1}^{(1)}, x_{m 1}^{(2)}, x_{m 1}^{(3)}, x_{m 1}^{(4)} & \cdots & x_{m n}^{(1)}, x_{m n}^{(2)}, x_{m n}^{(3)}, x_{m n}^{(4)}\end{array}\right), \\ \tilde{Y}=\left(\begin{array}{ccc}\left(y_{11}^{(1)}, y_{11}^{(2)}, y_{11}^{(3)}, y_{11}^{(4)}\right) & \cdots & \left(y_{1 n}^{(1)}, y_{1 n}^{(2)}, y_{1 n}^{(3)}, y_{1 n}^{(4)}\right) \\ \vdots & \ddots & \vdots \\ y_{m 1}^{(1)}, y_{m 1}^{(2)}, y_{m 1}^{(3)}, y_{m 1}^{(4)} & \cdots & y_{m n}^{(1)}, y_{m n}^{(2)}, y_{m n}^{(3)}, y_{m n}^{(4)}\end{array}\right) .\end{array}\right.$

In Section 3.3, the positive solution to the CTrFFSME is approximated numerically by extending the GI method in Theorem 1 and LSI method in Theorem 2.

3.3. Proposed Numerical Methods for Solving CTrFFSME. In this section, two numerical methods are developed: the fuzzy gradient iterative (FGI) method and the fuzzy least square iterative (FLSI) method. The details of the FGI methods are discussed as follows.

3.3.1. Fuzzy Gradient Iterative (FGI) Method for CTrFFSME. In this method, the CTrFFSME in equation (1) is solved numerically by extending the GI method for solving the crisp linear matrix equation $A X=B$ in Theorem 1 to the CTrFFSME in equation (1). The following steps summarize the methods:

Step 1. Decompose $\widetilde{A}, \widetilde{B}, \widetilde{C}, \widetilde{D}, \widetilde{E}, \widetilde{F}, \widetilde{X}$, and $\widetilde{Y}$ into $A^{(l)}$, $B^{(l)}, \quad C^{(l)}, D^{(l)}, E^{(l)}, F^{(l)}, X^{(l)}$, and $Y^{(l)}$ where $l=1,2,3,4$, respectively, and convert the CTrFFSME in equation (1) to the system of linear matrix equations in equation (24) using Theorem 3.

Step 2. Using the hierarchical identification principle and Remark 5, the system of CSME in equation (25) can be decomposed into two subsystems: for $1 \leq l \leq 4$,

$$
\begin{aligned}
& \xi_{1}^{(l)}=\left(\begin{array}{c}
E^{(l)}-Y^{(l)} B^{(l)} \\
F^{(l)}-Y^{(l)} D^{(l)}
\end{array}\right), \\
& \xi_{2}^{(l)}=\left(E^{(l)}-A^{(l)} X^{(l)} F^{(l)}-C^{(l)} X^{(l)}\right),
\end{aligned}
$$

where the iterative positive solution to the system of CSME in equation (25) is the average of the iterative solution for the subsystems. Let $\gamma_{l}=\left(\begin{array}{c}A^{(l)} \\ C^{(l)}\end{array}\right)$ and $\beta_{l}=\left(\begin{array}{ll}B^{(l)} D^{(l)}\end{array}\right)$.

From equations (25) and (35), the following can be obtained: for $1 \leq l \leq 4$,

$$
\begin{aligned}
& \xi_{2}^{(l)}=\gamma_{l} X^{(l)}, \\
& \xi_{1}^{(l)}=Y^{(l)} \beta_{l} .
\end{aligned}
$$


Step 1. Convert the CTrFFSME in equation (1) to four systems of linear matrix equations using Theorem 3 Step 2. Apply Vec-operator and Kronecker product on the systems obtained in Step 1

Step 3. Convert the obtained systems in Step 2 to a single system $P Q=U$

Step 4. Multiply both sides of the system obtained in Step 3 by $P^{-1}$

Step 5. Solve the system of matrix equations in Step 4 and write the positive fuzzy solution as follows:

Algorithm 1: Fuzzy matrix vectorization and Kronecker product algorithm for solving CTrFFSME

Step 3. The iterative positive solution to the system of equations in equations (36a) and (36b) can be obtained by the GI method in Theorem 1 as follows: for $1 \leq l \leq 4$, we have
Step 4. Substitute equations (35) into (37) as follows:

$$
\left\{\begin{array}{l}
\hat{x}_{l}(k)=\hat{x}_{l}(k-1)+\alpha_{l} \cdot\left(\gamma_{l}\right)^{T}\left(\left(\begin{array}{c}
E^{(l)}-\hat{y}_{l}(k-1) B^{(l)} \\
F^{(l)}-\hat{y}_{l}(k-1) D^{(l)}
\end{array}\right)-\left(\begin{array}{c}
A^{(l)} \\
C^{(l)}
\end{array}\right) \hat{x}^{(l)}(k-1)\right), \\
\hat{y}_{l}(k)=\hat{y}_{l}(k-1)+\alpha_{l} \cdot\left(\left(E^{(l)}-A^{(l)} \hat{x}_{l}(k-1) F^{(l)}-C^{(l)} \hat{x}_{l}(k-1)\right)-\hat{y}_{l}(k-1)\left(B^{(l)} D^{(l)}\right)\right)\left(\beta_{l}\right)^{T} .
\end{array}\right.
$$

The obtained algorithm in equation (38) can be written as follows: for $1 \leq l \leq 4$, we have

$$
\left\{\begin{array}{l}
\hat{x}_{l}(k)=\hat{x}_{l}(k-1)+\alpha_{l} \cdot\left(\gamma_{l}\right)^{T}\left(\begin{array}{c}
E^{(l)}-A^{(l)} \hat{x}_{l}(k-1)-\hat{y}_{l}(k-1) B^{(l)} \\
F^{(l)}-C^{(l)} \hat{x}_{l}(k-1)-\hat{y}_{l}(k-1) D^{(l)}
\end{array}\right), \\
\hat{y}_{l}(k)=\hat{y}_{l}(k-1)+\alpha_{l} \cdot\left(E^{(l)}-A^{(l)} \hat{x}_{l}(k-1)-\hat{y}_{l}(k-1) B^{(l)} F^{(l)}-C^{(l)} \hat{x}_{l}(k-1)-\hat{y}_{l}(k-1) D^{(l)}\right)\left(\beta_{l}\right)^{T} .
\end{array}\right.
$$

Let $r_{l}(k-1)=E^{(l)}-A^{(l)} \hat{x}_{l}(k-1)-\hat{y}_{l}(k-1) B^{(l)}$ and $s_{l}(k-1)=F^{(l)}-C^{(l)} \hat{x}_{l}(k-1)-\hat{y}_{l}(k-1) D^{(l)}$, then where the convergence factor (step size) is given by for $1 \leq l \leq 4$, the approximated solution in equation (39) can be written in reduced form as follows:

$$
\left\{\begin{array}{l}
\hat{x}_{l}(k)=\hat{x}_{l}(k-1)+\alpha_{l} \cdot\left(\gamma_{l}\right)^{T}\left(\begin{array}{c}
r_{l}(k-1) \\
s_{l}(k-1)
\end{array}\right) \\
\hat{y}_{l}(k)=\hat{y}_{l}(k-1)+\alpha_{l} \cdot\left(r_{l}(k-1) s_{l}(k-1)\right)\left(\beta_{l}\right)^{T}
\end{array}\right.
$$

$$
0<\alpha_{l}<\frac{2}{\lambda_{\max }\left[\left(A^{(l)}\right)^{T} A^{(l)}\right]+\lambda_{\max }\left[B^{(l)}\left(B^{(l)}\right)^{T}\right]+\lambda_{\max }\left[\left(C^{(l)}\right)^{T} C^{(l)}\right]+\lambda_{\max }\left[D^{(l)}\left(D^{(l)}\right)^{T}\right]}
$$


It can also be obtained as follows:

$$
0<\alpha_{l}<\frac{2}{\left\|A^{(l)}\right\|^{2}+\left\|B^{(l)}\right\|^{2}+\left\|C^{(l)}\right\|^{2}+\left\|D^{(l)}\right\|^{2}}=\varphi,
$$

where $\left\|A^{(l)}\right\|^{2}=\operatorname{tr}\left[A^{(l)} \cdot\left(A^{(l)}\right)^{T}\right]$.

At step $k$ - th of the iteration, the following relative error is considered:

$$
\delta^{l}(k)=\sqrt{\frac{\left\|\hat{x}_{l}(k)-\hat{x}_{l}(k-1)\right\|^{2}+\left\|\hat{y}_{l}(k)-\hat{y}_{l}(k-1)\right\|^{2}}{\left\|\hat{x}_{l}(k)\right\|^{2}+\left\|\hat{y}_{l}(k)\right\|^{2}}} .
$$

Step 5. By Definition 10, the approximated fuzzy solutions obtained by the previous step to the CTrFFSME in equation (1) can be written as follows:

$$
\left\{\begin{array}{l}
\hat{x}=\left(\hat{x}^{(1)}, \hat{x}^{(2)}, \hat{x}^{(3)}, \hat{x}^{(4)}\right) \\
\hat{y}=\left(\hat{y}^{(1)}, \hat{y}^{(2)}, \hat{y}^{(3)}, \hat{y}^{(4)}\right) .
\end{array}\right.
$$

It can also be written in matrix form as

$$
\left\{\begin{array}{c}
\hat{x}=\left(\begin{array}{ccc}
\left(\hat{x}_{11}^{(1)}, \hat{x}_{11}^{(2)}, \hat{x}_{11}^{(3)}, \hat{x}_{11}^{(4)}\right) & \ldots & \left(\hat{x}_{1 n}^{(1)}, \hat{x}_{1 n}^{(2)}, \hat{x}_{1 n}^{(3)}, \hat{x}_{1 n}^{(4)}\right) \\
\vdots & \ddots & \vdots \\
\hat{x}_{m 1}^{(1)}, \hat{x}_{m 1}^{(2)}, \hat{x}_{m 1}^{(3)}, \hat{x}_{m 1}^{(4)} & \ldots & \hat{x}_{m n}^{(1)}, \hat{x}_{m n}^{(2)}, \hat{x}_{m n}^{(3)}, \hat{x}_{m n}^{(4)}
\end{array}\right), \\
\hat{y}=\left(\begin{array}{ccc}
\left(\hat{y}_{11}^{(1)}, \hat{y}_{11}^{(2)}, \hat{y}_{11}^{(3)}, \hat{y}_{11}^{(4)}\right) & \ldots & \left(\hat{y}_{1 n}^{(1)}, \hat{y}_{1 n}^{(2)}, \hat{y}_{1 n}^{(3)}, \hat{y}_{1 n}^{(4)}\right) \\
\vdots & \ddots & \vdots \\
\hat{y}_{m 1}^{(1)}, \hat{y}_{m 1}^{(2)}, \hat{y}_{m 1}^{(3)}, \hat{y}_{m 1}^{(4)} & \ldots & \hat{y}_{m n}^{(1)}, \hat{y}_{m n}^{(2)}, \hat{y}_{m n}^{(3)}, \hat{y}_{m n}^{(4)}
\end{array}\right) .
\end{array}\right.
$$

In Theorem 4, we prove that the iterative solution obtained by the FGI method converges to the positive solution of the positive CTrFFSME for any initial value.

Theorem 4. If the system of CSME in equation (25) has a unique positive solution $\left(X^{(l)}, Y^{(l)}\right)$, then the iterative solution $\left(\hat{x}^{(l)}(k), \hat{y}^{(l)}(k)\right)$ in equation (43) converges to $\left(X^{(l)}, Y^{(l)}\right)$ for any initial values $\hat{x}^{(l)}(0), \hat{y}^{(l)}(0)$ for $1 \leq l \leq 4$ (i.e., if $k \longrightarrow \infty$, then $X^{(l)}=\hat{x}^{(l)}(k)$ and $Y^{(l)}=\hat{y}^{(l)}(k)$ ).

Proof. Let $\psi(k)$ be the error at each $k$, for $k=1, \ldots, n$ and $1 \leq l \leq 4$.

$$
\begin{gathered}
\psi(k)=\psi_{1}(k)+\psi_{2}(k), \\
\psi_{1}(k)=X^{(l)}-\hat{x}^{(l)}(k), \\
\psi_{2}(k)=Y^{(l)}-\hat{y}^{(l)}(k) .
\end{gathered}
$$

From equations (24), (39), (46a), and (46b), the following is obtained:

$$
\left\{\begin{array}{l}
\psi_{1}(k)=\psi_{1}(k-1)+\alpha_{l} \cdot\left(\begin{array}{c}
A^{(l)} \\
C^{(l)}
\end{array}\right)^{T}\left(\begin{array}{c}
-A^{(l)} \psi_{1}(k-1)-\psi_{2}(k-1) B^{(l)} \\
-C^{(l)} \psi_{1}(k-1)-\psi_{2}(k-1) D^{(l)}
\end{array}\right) \\
\psi_{2}(k)=\psi_{2}(k-1)+\alpha_{l} \cdot\left(-A^{(l)} \psi_{1}(k-1)-\psi_{2}(k-1) B^{(l)}-C^{(l)} \psi_{1}(k-1)-\psi_{2}(k-1) D^{(l)}\right)\left(B^{(l)} D^{(l)}\right)^{T} .
\end{array}\right.
$$

Taking $\|\cdot\|^{2}$ to both sides of equation (47) gives the following:

$$
\left\{\begin{array}{l}
\left\|\psi_{1}(k)\right\|^{2}=\left\|\psi_{1}(k-1)+\alpha_{l} \cdot\left(\begin{array}{c}
A^{(l)} \\
C^{(l)}
\end{array}\right)^{T}\left(\begin{array}{c}
-A^{(l)} \psi_{1}(k-1)-\psi_{2}(k-1) B^{(l)} \\
-C^{(l)} \psi_{1}(k-1)-\psi_{2}(k-1) D^{(l)}
\end{array}\right)\right\|^{2}, \\
\left\|\psi_{2}(k)\right\|^{2}=\left\|\psi_{2}(k-1)+\alpha_{l} \cdot\left(-A^{(l)} \psi_{1}(k-1)-\psi_{2}(k-1) B^{(l)}-C^{(l)} \psi_{1}(k-1)-\psi_{2}(k-1) D^{(l)}\right)\left(B^{(l)} D^{(l)}\right)^{T}\right\|^{2} .
\end{array}\right.
$$

Remark 8. The following steps in the proof are long; therefore, the system in equation (48) needs to be separated into two equations in the following steps of the proof.
It is not hard to prove that $\|A+B\|^{2}=\operatorname{tr}((A+$ $\left.B)^{T}(A+B)\right)=\|A\|^{2}+2 \operatorname{tr}\left(A^{T} B\right)+\|B\|^{2}$. Thus, equation $(48)$ can be written as 


$$
\begin{aligned}
\left\|\psi_{1}(k)\right\|^{2}= & \left\|\psi_{1}(k-1)\right\|^{2}+2 \alpha_{l} \operatorname{tr}\left[\psi_{1}^{T}(k-1)\left(\left(\begin{array}{c}
A^{(l)} \\
C^{(l)}
\end{array}\right)^{T}\left(\begin{array}{c}
-A^{(l)} \psi_{1}(k-1)-\psi_{2}(k-1) B^{(l)} \\
-C^{(l)} \psi_{1}(k-1)-\psi_{2}(k-1) D^{(l)}
\end{array}\right)\right)\right] \\
& +\alpha_{l}^{2}\left\|\left(\begin{array}{c}
A^{(l)} \\
C^{(l)}
\end{array}\right)^{T}\left(\begin{array}{c}
-A^{(l)} \psi_{1}(k-1)-\psi_{2}(k-1) B^{(l)} \\
-C^{(l)} \psi_{1}(k-1)-\psi_{2}(k-1) D^{(l)}
\end{array}\right)\right\|^{2},
\end{aligned}
$$

$$
\begin{aligned}
\left\|\psi_{2}(k)\right\|^{2}= & \left\|\psi_{2}(k-1)\right\|^{2}+2 \alpha_{l} \operatorname{tr}\left[\psi_{2}^{T}(k-1)\left(\left(-A^{(l)} \psi_{1}(k-1)-\psi_{2}(k-1) B^{(l)}-C^{(l)} \psi_{1}(k-1)-\psi_{2}(k-1) D^{(l)}\right)\left(B^{(l)} D^{(l)}\right)^{T}\right)\right] \\
& +\alpha_{l}^{2}\left\|\left(-A^{(l)} \psi_{1}(k-1)-\psi_{2}(k-1) B^{(l)}-C^{(l)} \psi_{1}(k-1)-\psi_{2}(k-1) D^{(l)}\right)\left(B^{(l)} D^{(l)}\right)^{T}\right\|^{2} .
\end{aligned}
$$

Applying norm properties and matrix multiplication gives

$$
\begin{aligned}
\left\|\psi_{1}(k)\right\|^{2} \leq & \left\|\psi_{1}(k-1)\right\|^{2}+2 \alpha_{l} \operatorname{tr}\left[\left(A^{(l)} \psi_{1}(k-1)\right)^{T}\left(-A^{(l)} \psi_{1}(k-1)-\psi_{2}(k-1) B^{(l)}\right)\right. \\
& \left.+\left(C^{(l)} \psi_{1}(k-1)\right)^{T}\left(-C^{(l)} \psi_{1}(k-1)-\psi_{2}(k-1) D^{(l)}\right)\right] \\
& +\alpha_{l}^{2}\left\|\left(\begin{array}{c}
A^{(l)} \\
C^{(l)}
\end{array}\right)^{T}\left(\begin{array}{l}
-A^{(l)} \psi_{1}(k-1)-\psi_{2}(k-1) B^{(l)} \\
-C^{(l)} \psi_{1}(k-1)-\psi_{2}(k-1) D^{(l)}
\end{array}\right)\right\|, \\
\left\|\psi_{2}(k)\right\|^{2} \leq & \left\|\psi_{2}(k-1)\right\|^{2}+2 \alpha_{l} \operatorname{tr}\left[\left(\psi_{2}(k-1) B^{(l)}\right)^{T}\left(-A^{(l)} \psi_{1}(k-1)-\psi_{2}(k-1) B^{(l)}\right)\right. \\
& \left.+\left(\psi_{2}(k-1) D^{(l)}\right)^{T}\left(-C^{(l)} \psi_{1}(k-1)-\psi_{2}(k-1) D^{(l)}\right)\right] \\
& +\alpha_{l}^{2}\left\|\left(-A^{(l)} \psi_{1}(k-1)-\psi_{2}(k-1) B^{(l)}-C^{(l)} \psi_{1}(k-1)-\psi_{2}(k-1) D^{(l)}\right)\left(B^{(l)} D^{(l)}\right)^{T}\right\|^{2} .
\end{aligned}
$$

Applying norm properties on equations (50a) and (50b) gives

$$
\begin{aligned}
\left\|\psi_{1}(k)\right\|^{2} \leq & \left\|\psi_{1}(k-1)\right\|^{2}+2 \alpha_{l} \operatorname{tr}\left[\left(A^{(l)} \psi_{1}(k-1)\right)^{T}\left(-A^{(l)} \psi_{1}(k-1)-\psi_{2}(k-1) B^{(l)}\right)\right. \\
& \left.+\left(C^{(l)} \psi_{1}(k-1)\right)^{T}\left(-C^{(l)} \psi_{1}(k-1)-\psi_{2}(k-1) D^{(l)}\right)\right] \\
& +\alpha_{l}^{2}\left(\left\|A^{(l)}\right\|^{2}+\left\|C^{(l)}\right\|^{2}\right)\left[\left\|-A^{(l)} \psi_{1}(k-1)-\psi_{2}(k-1) B^{(l)}\right\|^{2}+\left\|-C^{(l)} \psi_{1}(k-1)-\psi_{2}(k-1) D^{(l)}\right\|^{2}\right], \\
\left\|\psi_{2}(k)\right\|^{2} \leq & \left\|\psi_{2}(k-1)\right\|^{2}+2 \alpha_{l} \operatorname{tr}\left[\left(\psi_{2}(k-1) B^{(l)}\right)^{T}\left(-A^{(l)} \psi_{1}(k-1)-\psi_{2}(k-1) B^{(l)}\right)\right. \\
& \left.+\left(\psi_{2}(k-1) D^{(l)}\right)^{T}\left(-C^{(l)} \psi_{1}(k-1)-\psi_{2}(k-1) D^{(l)}\right)\right] \\
& +\alpha_{l}^{2}\left(\left\|B^{(l)}\right\|^{2}+\left\|D^{(l)}\right\|^{2}\right)\left[\left\|-A^{(l)} \psi_{1}(k-1)-\psi_{2}(k-1) B^{(l)}\right\|^{2}+\left\|-C^{(l)} \psi_{1}(k-1)-\psi_{2}(k-1) D^{(l)}\right\|^{2}\right] .
\end{aligned}
$$


By the definition of the error in equation (45) and by equations (51a) and (51b), the following is obtained:

$$
\begin{aligned}
\|\psi(k)\|^{2} \leq & \left\|\psi_{1}(k-1)\right\|^{2}+2 \alpha_{l} \operatorname{tr}\left[\left(A^{(l)} \psi_{1}(k-1)\right)^{T}\left(-A^{(l)} \psi_{1}(k-1)-\psi_{2}(k-1) B^{(l)}\right)\right. \\
& \left.+\left(C^{(l)} \psi_{1}(k-1)\right)^{T}\left(-C^{(l)} \psi_{1}(k-1)-\psi_{2}(k-1) D^{(l)}\right)\right] \\
& +\alpha_{l}^{2}\left(\left\|A^{(l)}\right\|^{2}+\left\|C^{(l)}\right\|^{2}\right)\left[\left\|-A^{(l)} \psi_{1}(k-1)-\psi_{2}(k-1) B^{(l)}\right\|^{2}+\left\|-C^{(l)} \psi_{1}(k-1)-\psi_{2}(k-1) D^{(l)}\right\|^{2}\right] \\
& +\left\|\psi_{2}(k-1)\right\|^{2}+2 \alpha_{l} \operatorname{tr}\left[\left(\psi_{2}(k-1) B^{(l)}\right)^{T}\left(-A^{(l)} \psi_{1}(k-1)-\psi_{2}(k-1) B^{(l)}\right)\right. \\
& \left.+\left(\psi_{2}(k-1) D^{(l)}\right)^{T}\left(-C^{(l)} \psi_{1}(k-1)-\psi_{2}(k-1) D^{(l)}\right)\right] \\
& +\alpha_{l}^{2}\left(\left\|B^{(l)}\right\|^{2}+\left\|D^{(l)}\right\|^{2}\right)\left[\left\|-A^{(l)} \psi_{1}(k-1)-\psi_{2}(k-1) B^{(l)}\right\|^{2}+\left\|-C^{(l)} \psi_{1}(k-1)-\psi_{2}(k-1) D^{(l)}\right\|^{2}\right],
\end{aligned}
$$

which can be written as

$$
\begin{aligned}
\|\psi(k)\|^{2} \leq & \left\|\psi_{1}(k-1)\right\|^{2}+\left\|\psi_{2}(k-1)\right\|^{2}+2 \alpha_{l} \operatorname{tr}\left[\left(A^{(l)} \psi_{1}(k-1)\right)^{T}\left(-A^{(l)} \psi_{1}(k-1)-\psi_{2}(k-1) B^{(l)}\right)\right. \\
& \left.+\left(C^{(l)} \psi_{1}(k-1)\right)^{T}\left(-C^{(l)} \psi_{1}(k-1)-\psi_{2}(k-1) D^{(l)}\right)\right] \\
& +\alpha_{l}^{2}\left(\left\|A^{(l)}\right\|^{2}+\left\|C^{(l)}\right\|^{2}\right)\left[\left\|-A^{(l)} \psi_{1}(k-1)-\psi_{2}(k-1) B^{(l)}\right\|^{2}+\left\|-C^{(l)} \psi_{1}(k-1)-\psi_{2}(k-1) D^{(l)}\right\|^{2}\right] \\
& +2 \alpha_{l} \operatorname{tr}\left[\left(\psi_{2}(k-1) B^{(l)}\right)^{T}\left(-A^{(l)} \psi_{1}(k-1)-\psi_{2}(k-1) B^{(l)}\right)+\left(\psi_{2}(k-1) D^{(l)}\right)^{T}\left(-C^{(l)} \psi_{1}(k-1)-\psi_{2}(k-1) D^{(l)}\right)\right] \\
& +\alpha_{l}^{2}\left(\left\|B^{(l)}\right\|^{2}+\left\|D^{(l)}\right\|^{2}\right)\left[\left\|-A^{(l)} \psi_{1}(k-1)-\psi_{2}(k-1) B^{(l)}\right\|^{2}+\left\|-C^{(l)} \psi_{1}(k-1)-\psi_{2}(k-1) D^{(l)}\right\|^{2}\right],
\end{aligned}
$$

$\|\psi(k)\|^{2} \leq\left\|\psi_{1}(k-1)\right\|^{2}+\left\|\psi_{2}(k-1)\right\|^{2}+2 \alpha_{l} \operatorname{tr}\left[\left(A^{(l)} \psi_{1}(k-1)+\psi_{2}(k-1) B^{(l)}\right)^{T}\left(-A^{(l)} \psi_{1}(k-1)-\psi_{2}(k-1) B^{(l)}\right)\right.$

$$
\begin{aligned}
& \left.+\left(C^{(l)} \psi_{1}(k-1)+\psi_{2}(k-1) D^{(l)}\right)^{T}\left(-C^{(l)} \psi_{1}(k-1)-\psi_{2}(k-1) D^{(l)}\right)\right] \\
& +\alpha_{l}^{2}\left(\left\|A^{(l)}\right\|^{2}+\left\|C^{(l)}\right\|^{2}+\left\|B^{(l)}\right\|^{2}+\left\|D^{(l)}\right\|^{2}\right)\left[\left\|-A^{(l)} \psi_{1}(k-1)-\psi_{2}(k-1) B^{(l)}\right\|^{2}+\left\|-C^{(l)} \psi_{1}(k-1)-\psi_{2}(k-1) D^{(l)}\right\|^{2}\right],
\end{aligned}
$$

$\|\psi(k)\|^{2} \leq\left\|\psi_{1}(k-1)\right\|^{2}+\left\|\psi_{2}(k-1)\right\|^{2}-2 \alpha_{l} \operatorname{tr}\left[\left(A^{(l)} \psi_{1}(k-1)+\psi_{2}(k-1) B^{(l)}\right)^{T}\left(A^{(l)} \psi_{1}(k-1)+\psi_{2}(k-1) B^{(l)}\right)\right.$

$$
\begin{aligned}
& \left.+\left(C^{(l)} \psi_{1}(k-1)+\psi_{2}(k-1) D^{(l)}\right)^{T}\left(C^{(l)} \psi_{1}(k-1)+\psi_{2}(k-1) D^{(l)}\right)\right] \\
& +\alpha_{l}^{2}\left(\left\|A^{(l)}\right\|^{2}+\left\|C^{(l)}\right\|^{2}+\left\|B^{(l)}\right\|^{2}+\left\|D^{(l)}\right\|^{2}\right)\left[\left\|A^{(l)} \psi_{1}(k-1)+\psi_{2}(k-1) B^{(l)}\right\|^{2}+\left\|C^{(l)} \psi_{1}(k-1)+\psi_{2}(k-1) D^{(l)}\right\|^{2}\right],
\end{aligned}
$$

$\|\psi(k)\|^{2} \leq\|\psi(k-1)\|^{2}-2 \alpha_{l}\left[\left\|A^{(l)} \psi_{1}(k-1)+\psi_{2}(k-1) B^{(l)}\right\|^{2}+\left\|C^{(l)} \psi_{1}(k-1)+\psi_{2}(k-1) D^{(l)}\right\|^{2}\right]$

$$
+\frac{2 \alpha_{l}^{2}}{\varphi}\left[\left\|A^{(l)} \psi_{1}(k-1)+\psi_{2}(k-1) B^{(l)}\right\|^{2}+\left\|C^{(l)} \psi_{1}(k-1)+\psi_{2}(k-1) D^{(l)}\right\|^{2}\right] .
$$


By equation (41b), the following can be obtained:

$$
\begin{aligned}
\|\psi(k)\|^{2} \leq & \|\psi(k-1)\|^{2}-2 \alpha_{l}\left(1-\frac{\alpha_{l}}{\varphi}\right)\left[\left\|A^{(l)} \psi_{1}(k-1)+\psi_{2}(k-1) B^{(l)}\right\|^{2}\right. \\
& \left.+\left\|C^{(l)} \psi_{1}(k-1)+\psi_{2}(k-1) D^{(l)}\right\|^{2}\right] .
\end{aligned}
$$

At $\quad k=1, \quad\|\psi(1)\|^{2} \leq\|\psi(0)\|^{2}-2 \alpha_{l} \quad\left(1-\left(\alpha_{l} /\right.\right.$ $\varphi))\left[\left\|A^{(l)} \psi_{1}(0)+\psi_{2}(0) B^{(l)}\right\|^{2}+\left\|C^{(l)} \psi_{1}(0)+\psi_{2}(0) D^{(l)}\right\|^{2}\right]$.

At $\quad k=2, \quad\|\psi(2)\|^{2} \leq\|\psi(1)\|^{2}-2 \alpha_{l}\left(1-\left(\alpha_{l} / \varphi\right)\right)$ $\left[\left\|A^{(l)} \psi_{1}(1)+\psi_{2}(1) B^{(l)}\right\|^{2}+\left\|C^{(l)} \psi_{1}(0)+\psi_{2}(0) D^{(l)}\right\|^{2}\right]$.

At $k=3,\|\psi(3)\|^{2} \leq\|\psi(2)\|^{2}-2 \alpha_{l}\left(1-\left(\alpha_{l} / \varphi\right)\right) \quad\left[\| A^{(l)} \psi_{1}\right.$ (2) $\left.+\psi_{2}(2) B^{(l)}\left\|^{2}+\right\| C^{(l)} \psi_{1}(2)+\psi_{2}(2) D^{(l)} \|^{2}\right]$.

At $k=n-1,\|\psi(n-1)\|^{2} \leq\|\psi(n-2)\|^{2}-2 \alpha_{l}\left(1-\left(\alpha_{l} / \varphi\right)\right)$ $\left[\left\|A^{(l)} \psi_{1}(n-2)+\psi_{2}(n-2) \quad B^{(l)}\right\|^{2}+\| C^{(l)} \psi_{1}(n-2)+\psi_{2}\right.$ $\left.(n-2) D^{(l)} \|^{2}\right]$.

At $k=n,\|\psi(n)\|^{2} \leq\|\psi(n-1)\|^{2}-2 \alpha_{l}\left(1-\left(\alpha_{l} / \varphi\right)\right)\left[\| A^{(l)}\right.$ $\psi_{1}(n-1)+\psi_{2}(n-1) B^{(l)}\left\|^{2}+\right\| C^{(l)} \psi_{1}(n-1)+\psi_{2}(n-1)$ $\left.D^{(l)} \|^{2}\right]$.

Therefore, the following is obtained:

$$
\begin{aligned}
\|\psi(k)\|^{2} \leq & \|\psi(k-1)\|^{2}-2 \alpha_{l}\left(1-\frac{\alpha_{l}}{\varphi}\right)\left[\left\|A^{(l)} \psi_{1}(k-1)+\psi_{2}(k-1) B^{(l)}\right\|^{2}\right. \\
& \left.+\left\|C^{(l)} \psi_{1}(k-1)+\psi_{2}(k-1) D^{(l)}\right\|^{2}\right] .
\end{aligned}
$$

If the convergence factor $\alpha$ is chosen to satisfy equation (41b) and $k \longrightarrow \infty$, then

$$
\sum_{k=1}^{\infty}\left(\left\|A^{(l)} \psi_{1}(k)+\psi_{2}(k) B^{(l)}\right\|^{2}+\left\|C^{(l)} \psi_{1}(k)+\psi_{2}(k) D^{(l)}\right\|^{2}\right)<\infty .
$$

Therefore,

$$
\begin{aligned}
& \lim _{k \longrightarrow \infty}\left(A^{(l)} \psi_{1}(k)+\psi_{2}(k) B^{(l)}\right)=0, \\
& \lim _{k \rightarrow \infty}\left(C^{(l)} \psi_{1}(k)+\psi_{2}(k) D^{(l)}\right)=0 .
\end{aligned}
$$

Since $A^{(l)}>0, B^{(l)}>0, C^{(l)}>0$, and $D^{(l)}>0$, then

$$
\begin{aligned}
& \lim _{k \longrightarrow \infty} \psi_{1}(k)=0, \\
& \lim _{k \longrightarrow \infty} \psi_{2}(k)=0 .
\end{aligned}
$$

By equations (46a) and (46b), the following is obtained:

$$
\begin{aligned}
& \lim _{k \longrightarrow \infty}\left(X^{(l)}-\hat{x}^{(l)}(k)\right)=0, \\
& \lim _{k \rightarrow \infty}\left(Y^{(l)}-\hat{y}^{(l)}(k)\right)=0 .
\end{aligned}
$$

Consequently, if $k \longrightarrow \infty$, then $X^{(l)}=\hat{x}^{(l)}(k)$ and $Y^{(l)}=\hat{y}^{(l)}(k)$

Therefore, if the system of CSME in equation (25) has a unique positive solution $\left(X^{(l)}, Y^{(l)}\right)$, then the iterative solution $\left(\hat{x}^{(l)}(k), \hat{y}^{(l)}(k)\right)$ in equation (43) converges to $\left(X^{(l)}, Y^{(l)}\right)$ for any initial values $\hat{x}^{(l)}(0), \hat{y}^{(l)}(0)$ for $1 \leq l \leq 4$ (i.e., if $k \longrightarrow \infty$, then $X^{(l)}=\hat{x}^{(l)}(k)$ and $Y^{(l)}=\hat{y}^{(l)}(k)$ ).

The FGI algorithm is shown as Algorithm 2. This algorithm can be used by different software for solving the CTrFFSME in equation (1).

In the following method, the LSI method in Theorem 2 is extended and applied for solving the CTrFFSME in equation (1).

3.3.2. Fuzzy Least-Square Iterative (FLSI) Method for CTrFFSME. In this method, the least square iterative method in Theorem 2 for solving $A X=B$ is applied for the CTrFFSME in equation (1). The first two steps of this method are similar to those of the fuzzy gradient iterative method.

Step 3. The iterative positive solution to the system of equations in equations (36a) and (36b) can be obtained by the LSI method in Theorem 2 as follows: for $1 \leq l \leq 4$, we have

$$
\left\{\begin{array}{l}
\hat{x}_{l}(k)=\hat{x}_{l}(k-1)+\alpha_{l}\left(\left(\begin{array}{c}
A^{(l)} \\
C^{(l)}
\end{array}\right)^{T}\left(\begin{array}{c}
A^{(l)} \\
C^{(l)}
\end{array}\right)\right)^{-1}\left(\begin{array}{c}
A^{(l)} \\
C^{(l)}
\end{array}\right)^{T}\left(\begin{array}{c}
E^{(l)}-A^{(l)} \hat{x}_{l}(k-1)-\hat{y}_{l}(k-1) B^{(l)} \\
F^{(l)}-C^{(l)} \hat{x}_{l}(k-1)-\hat{y}_{l}(k-1) D^{(l)}
\end{array}\right), \\
\hat{y}_{l}(k)=\hat{y}_{l}(k-1)+\alpha_{l} \cdot\left(E^{(l)}-A^{(l)} \hat{x}_{l}(k-1)-\hat{y}_{l}(k-1) B^{(l)} F^{(l)}-C^{(l)} \hat{x}_{l}(k-1)-\hat{y}_{l}(k-1) D^{(l)}\right) \\
\left(B^{(l)} D^{(l)}\right)^{T}\left(\left(\begin{array}{ll}
B^{(l)} & \left.\left.D^{(l)}\right)\left(B^{(l)} D^{(l)}\right)^{T}\right)^{-1} .
\end{array}\right.\right.
\end{array}\right.
$$

Step 4. Let $\gamma_{l}=\left(\begin{array}{l}A^{(l)} \\ C^{(l)}\end{array}\right), \beta_{l}=\left(\begin{array}{ll}B^{(l)} & D^{(l)}\end{array}\right), r_{l}(k-1)=$ $E^{(l)}-A^{(l)} \hat{x}_{l}(k-1)-\hat{y}_{l}(k-1) B^{(l)}$, and $s_{l}(k-1)=F^{(l)}$
$-C^{(l)} \hat{x}_{l}(k-1)-\hat{y}_{l}(k-1) D^{(l)}$. For $1 \leq l \leq 4$, the approximated fuzzy solution in equation (60) can be written as follows: 
Input that $\widetilde{A}, \widetilde{B}, \widetilde{C}, \widetilde{D}, \widetilde{E}, \widetilde{F}$ \# Split each matrix into four matrices (e.g., $A^{(1)}, A^{(2)}, A^{(3)}, A^{(4)}$ )

for $l=1,2,3,4$

Choose $\alpha_{l}, \varepsilon, \hat{x}_{l}(0)=0, \hat{y}_{l}(0)=0 \# 0$ is the Zero matrix with the same dimension as $X^{(l)}(k)$ and $Y^{(l)}(k)$.

While $k=0,1,2, \ldots, n$ do

$$
\begin{aligned}
& \left\{\begin{array}{l}
\hat{x}_{l}(k)=\hat{x}_{l}(k-1)+\alpha_{l} \cdot\left(\gamma_{l}\right)^{T}\left(\begin{array}{c}
r_{l}(k-1) \\
s_{l}(k-1)
\end{array}\right) \\
\hat{y}_{l}(k)=\hat{y}_{l}(k-1)+\alpha_{l} \cdot\left(r_{l}(k-1) s_{l}(k-1)\right)\left(\beta_{l}\right)^{T}
\end{array}\right. \\
& r_{l}(k-1)=E^{(l)}-A^{(l)} \hat{x}_{l}(k-1)-\hat{y}_{l}(k-1) B^{(l)} \\
& s_{l}(k-1)=F^{(l)}-C^{(l)} \hat{x}_{l}(k-1)-\hat{y}_{l}(k-1) D^{(l)} \\
& \gamma_{l}=\left(\begin{array}{c}
a_{i j}^{(l)} \\
c_{i j}^{(l)}
\end{array}\right), \beta_{l}=\left(\begin{array}{ll}
b_{i j}^{(l)} & d_{i j}^{(l)}
\end{array}\right) \\
& \delta_{l}(k)=\sqrt{\left(\left(\left\|\hat{x}_{l}(k)-\hat{x}_{l}(k-1)\right\|^{2}+\left\|\hat{y}_{l}(k)-\hat{y}_{l}(k-1)\right\|^{2}\right) /\left(\left\|\hat{x}_{l}(k)\right\|^{2}+\left\|\hat{y}_{l}(k)\right\|^{2}\right)\right)} \\
& \text { If } \delta_{l}(k)<\varepsilon \text { then } \\
& \text { print }\left(\hat{x}_{l}(k), \hat{y}_{l}(k)\right) \text {; } \\
& \text { print ("number of iterations }=", k \text { ). } \\
& \text { else } \\
& \left\{\begin{array}{l}
\hat{x}_{l}(k)=\hat{x}_{l}(k-1)+\alpha_{l} \cdot\left(\gamma_{l}\right)^{T}\left(\begin{array}{l}
r_{l}(k-1) \\
s_{l}(k-1)
\end{array}\right) \\
\hat{y}_{l}(k)=\hat{y}_{l}(k-1)+\alpha_{l} \cdot\left(r_{l}(k-1) s_{l}(k-1)\right)\left(\beta_{l}\right)^{T}
\end{array},\right. \\
& \text { update } k \text {. } \\
& k=k+1 \\
& \text { end } \\
& \text { print }\left(\hat{x}_{l}(k), \hat{y}_{l}(k)\right) \text {; } \\
& \text { print }(\text { "number of iterations }=", k) \text {. }
\end{aligned}
$$$$
\text { end }
$$

Algorithm 2: FGI algorithm for CTrFFSME

$$
\left\{\begin{array}{l}
\hat{x}_{l}(k)=\hat{x}_{l}(k-1)+\alpha_{l}\left(\left(\gamma_{l}\right)^{T} \gamma_{l}\right)^{-1}\left(\gamma_{l}\right)^{T}\left(\begin{array}{c}
r_{l}(k-1) \\
s_{l}(k-1)
\end{array}\right), \\
\hat{y}_{l}(k)=\hat{y}_{l}(k-1)+\alpha_{l} \cdot\left(r_{l}(k-1) s_{l}(k-1)\right)\left(\beta_{l}\right)^{T}\left(\left(\beta_{l}\right)^{T} \beta_{l}\right)^{-1},
\end{array}\right.
$$

where the convergence factor (step size) is given by

$$
0<\alpha_{l}<\frac{2}{\lambda_{\max }\left[\alpha_{l}\left(\left(\alpha_{l}\right)^{T} \alpha_{l}\right)^{-1}\left(\alpha_{l}\right)^{T}\right]+\lambda_{\max }\left[\left(\beta_{l}\right)^{T}\left(\left(\beta_{l}\right)^{T} \beta_{l}\right)^{-1} \beta_{l}\right]}=\frac{2}{\varphi_{1}+\varphi_{2}} .
$$

At step $k$ - th of the iteration, the following relative error is considered:

$$
\delta^{l}(k)=\sqrt{\frac{\left\|\hat{x}_{l}(k)-\hat{x}_{l}(k-1)\right\|^{2}+\left\|\hat{y}_{l}(k)-\hat{y}_{l}(k-1)\right\|^{2}}{\left\|\hat{x}_{l}(k)\right\|^{2}+\left\|\hat{y}_{l}(k)\right\|^{2}}}
$$

Step 5. By Definition 10, the approximated fuzzy solutions obtained by the previous step to the CTrFFSME in equation (1) can be written as follows:

$$
\left\{\begin{array}{l}
\hat{x}=\left(\hat{x}^{(1)}, \hat{x}^{(2)}, \hat{x}^{(3)}, \hat{x}^{(4)}\right) \\
\hat{y}=\left(\hat{y}^{(1)}, \hat{y}^{(2)}, \hat{y}^{(3)}, \hat{y}^{(4)}\right)
\end{array}\right.
$$


It can also be written in matrix form as

$$
\left\{\begin{array}{c}
\hat{x}=\left(\begin{array}{ccc}
\left(\hat{x}_{11}^{(1)}, \hat{x}_{11}^{(2)}, \hat{x}_{11}^{(3)}, \hat{x}_{11}^{(4)}\right) & \ldots & \left(\hat{x}_{1 n}^{(1)}, \hat{x}_{1 n}^{(2)}, \hat{x}_{1 n}^{(3)}, \hat{x}_{1 n}^{(4)}\right) \\
\vdots & \ddots & \vdots \\
\left(\wedge^{(1)} \hat{x}^{(2)}, \hat{x}_{m 1}^{(3)}, \hat{x}_{m 1}^{(4)}\right) & \ldots & \left(\hat{x}_{m n}^{(1)}, \hat{x}_{m n}^{(2)}, \hat{x}_{m n}^{(3)}, \hat{x}_{m n}^{(4)}\right)
\end{array}\right), \\
\hat{y}=\left(\begin{array}{ccc}
\left(\hat{y}_{11}^{(1)}, \hat{y}_{11}^{(2)}, \hat{y}_{11}^{(3)}, \hat{y}_{11}^{(4)}\right) & \ldots & \left(\hat{y}_{1 n}^{(1)}, \hat{y}_{1 n}^{(2)}, \hat{y}_{1 n}^{(3)}, \hat{y}_{1 n}^{(4)}\right) \\
\vdots & \ddots & \vdots \\
\left(\hat{y}_{m 1}^{(1)}, \hat{y}_{m 1}^{(2)}, \hat{y}_{m 1}^{(3)}, \hat{y}_{m 1}^{(4)}\right) & \ldots & \left(\hat{y}_{m n}^{(1)}, \hat{y}_{m n}^{(2)}, \hat{y}_{m n}^{(3)}, \hat{y}_{m n}^{(4)}\right)
\end{array}\right) .
\end{array}\right.
$$

In Theorem 5, we prove that the iterative solution obtained by the FLSI method converges to the positive solution of the positive CTrFFSME for any initial value.

Theorem 5. If the system of CSME in equation (25) has a unique positive solution $\left(X^{(l)}, Y^{(l)}\right)$, then the iterative solution $\left(\hat{x}^{(l)}(k), \hat{y}^{(l)}(k)\right)$ in equation $\left(\phi_{\ell}\right)$ converges to $\left(X^{(l)}, Y^{(l)}\right)$ for any initial values $\hat{X}^{(0), \hat{y}}(0)$ for $_{\Lambda^{\prime}} 1 \leq l \leq 4$

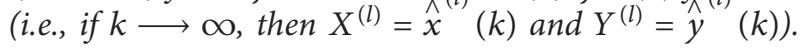

Proof. Let $\psi(k)$ be the error at each $k$, for $k=1, \ldots, n$ and $1 \leq l \leq 4$.

$$
\psi(k)=\left(\begin{array}{c}
A^{(l)} \\
C^{(l)}
\end{array}\right) \psi_{1}(k)+\psi_{2}(k)\left(B^{(l)} D^{(l)}\right),
$$

where

$$
\begin{aligned}
& \psi_{1}(k)=X^{(l)}-\hat{x}^{(l)}(k), \\
& \psi_{2}(k)=Y^{(l)}-\hat{y}^{(l)}(k) .
\end{aligned}
$$

From equations (24), (60), (67a), and (67b), the following is obtained:

$$
\left\{\begin{array}{l}
\psi_{1}(k)=\psi_{1}(k-1)+\alpha_{l} \cdot\left(\left(\begin{array}{l}
A^{(l)} \\
C^{(l)}
\end{array}\right)^{T}\left(\begin{array}{l}
A^{(l)} \\
C^{(l)}
\end{array}\right)\right)^{-1}\left(\begin{array}{c}
A^{(l)} \\
C^{(l)}
\end{array}\right)^{T}\left(\begin{array}{c}
-A^{(l)} \psi_{1}(k-1)-\psi_{2}(k-1) B^{(l)} \\
-C^{(l)} \psi_{1}(k-1)-\psi_{2}(k-1) D^{(l)}
\end{array}\right) \\
\psi_{2}(k)=\psi_{2}(k-1)+\alpha_{l} \cdot\left(-A^{(l)} \psi_{1}(k-1)-\psi_{2}(k-1) B^{(l)}-C^{(l)} \psi_{1}(k-1)-\psi_{2}(k-1) D^{(l)}\right) \\
\left(B^{(l)} D^{(l)}\right)^{T}\left(\left(B^{(l)} D^{(l)}\right)\left(B^{(l)} D^{(l)}\right)^{T}\right)^{-1}
\end{array}\right.
$$

Taking $\|\cdot\|^{2}$ to both sides of equation (68) gives the following:

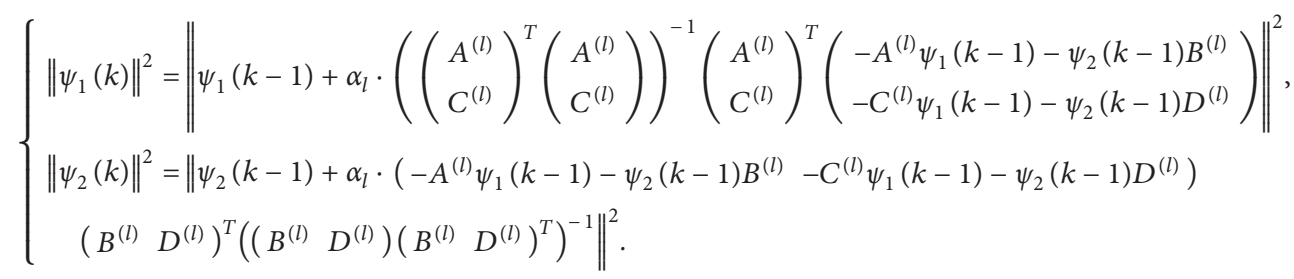

The following steps in the proof are long; therefore, the system in equation (69) has to be into two equations in the following steps of the proof.
Apply the following formula to equation (69) we get

$$
\begin{aligned}
\left\|A\left(X+\left((A)^{T} \cdot A\right)^{-1} Y\right)\right\|^{2}= & \operatorname{tr}\left(\left(A\left(X+\left((A)^{T} \cdot A\right)^{-1} Y\right)\right)^{T}\left(A\left(X+\left((A)^{T} \cdot A\right)^{-1} Y\right)\right)\right)=\|A X\|^{2}+2 \operatorname{tr}\left(X^{T} Y\right)+\left\|\left((A)^{T} \cdot A\right)^{-1} Y\right\|^{2} \\
\left\|\left(\begin{array}{l}
A^{(l)} \\
C^{(l)}
\end{array}\right) \psi_{1}(k)\right\|^{2}= & \left\|\left(\begin{array}{l}
A^{(l)} \\
C^{(l)}
\end{array}\right) \psi_{1}(k-1)\right\|^{2}+2 \alpha_{l} \operatorname{tr}\left[\psi_{1}^{T}(k-1)\left(\left(\begin{array}{c}
A^{(l)} \\
C^{(l)}
\end{array}\right)^{T}\left(\begin{array}{c}
-A^{(l)} \psi_{1}(k-1)-\psi_{2}(k-1) B^{(l)} \\
-C^{(l)} \psi_{1}(k-1)-\psi_{2}(k-1) D^{(l)}
\end{array}\right)\right)\right] \\
& +\alpha_{l}^{2}\left\|\left(\left(\begin{array}{l}
A^{(l)} \\
C^{(l)}
\end{array}\right)^{T}\left(\begin{array}{l}
A^{(l)} \\
C^{(l)}
\end{array}\right)\right)^{-1}\left(\begin{array}{l}
A^{(l)} \\
C^{(l)}
\end{array}\right)^{T}\left(\begin{array}{l}
-A^{(l)} \psi_{1}(k-1)-\psi_{2}(k-1) B^{(l)} \\
-C^{(l)} \psi_{1}(k-1)-\psi_{2}(k-1) D^{(l)}
\end{array}\right)\right\|^{2}
\end{aligned}
$$




$$
\begin{aligned}
& \left\|\psi_{2}(k)\left(B^{(l)} D^{(l)}\right)\right\|^{2}=\left\|\psi_{2}(k-1)\left(B^{(l)} D^{(l)}\right)\right\|^{2}+2 \alpha_{l} \operatorname{tr}\left[\psi_{2}^{T}(k-1)\right. \\
& \left.\cdot\left(\left(-A^{(l)} \psi_{1}(k-1)-\psi_{2}(k-1) B^{(l)}-C^{(l)} \psi_{1}(k-1)-\psi_{2}(k-1) D^{(l)}\right)\left(B^{(l)} D^{(l)}\right)^{T}\right)\right] \\
& +\alpha_{l}^{2} \|\left(-A^{(l)} \psi_{1}(k-1)-\psi_{2}(k-1) B^{(l)}-C^{(l)} \psi_{1}(k-1)-\psi_{2}(k-1) D^{(l)}\right) \\
& \cdot\left(B^{(l)} D^{(l)}\right)^{T}\left(\left(\begin{array}{ll}
B^{(l)} & D^{(l)}
\end{array}\right)\left(B^{(l)} D^{(l)}\right)^{T}\right)^{-1} \|^{2} \text {. }
\end{aligned}
$$

Applying norm properties and matrix multiplication gives

$$
\begin{aligned}
\left\|\left(\begin{array}{c}
A^{(l)} \\
C^{(l)}
\end{array}\right) \psi_{1}(k)\right\|^{2} \leq & \left\|\left(\begin{array}{c}
A^{(l)} \\
C^{(l)}
\end{array}\right) \psi_{1}(k-1)\right\|^{2}+2 \alpha_{l} \operatorname{tr}\left[\left(A^{(l)} \psi_{1}(k-1)\right)^{T}\left(-A^{(l)} \psi_{1}(k-1)-\psi_{2}(k-1) B^{(l)}\right)\right. \\
& \left.+\left(C^{(l)} \psi_{1}(k-1)\right)^{T}\left(-C^{(l)} \psi_{1}(k-1)-\psi_{2}(k-1) D^{(l)}\right)\right] \\
& +\alpha_{l}^{2}\left\|\left(\left(\begin{array}{l}
A^{(l)} \\
C^{(l)}
\end{array}\right)^{T}\left(\begin{array}{l}
A^{(l)} \\
C^{(l)}
\end{array}\right)\right)^{-1}\left(\begin{array}{l}
A^{(l)} \\
C^{(l)}
\end{array}\right)^{T}\left(\begin{array}{l}
-A^{(l)} \psi_{1}(k-1)-\psi_{2}(k-1) B^{(l)} \\
-C^{(l)} \psi_{1}(k-1)-\psi_{2}(k-1) D^{(l)}
\end{array}\right)\right\|^{2},
\end{aligned}
$$

$\left\|\psi_{2}(k)\left(B^{(l)} D^{(l)}\right)\right\|^{2} \leq\left\|\psi_{2}(k-1)\left(B^{(l)} D^{(l)}\right)\right\|^{2}+2 \alpha_{l} \operatorname{tr}\left[\left(\psi_{2}(k-1) B^{(l)}\right)^{T}\left(-A^{(l)} \psi_{1}(k-1)-\psi_{2}(k-1) B^{(l)}\right)\right.$

$$
\begin{aligned}
& \left.+\left(\psi_{2}(k-1) D^{(l)}\right)^{T}\left(-C^{(l)} \psi_{1}(k-1)-\psi_{2}(k-1) D^{(l)}\right)\right] \\
& +\alpha_{l}^{2} \|\left(-A^{(l)} \psi_{1}(k-1)-\psi_{2}(k-1) B^{(l)}-C^{(l)} \psi_{1}(k-1)-\psi_{2}(k-1) D^{(l)}\right) \\
& \cdot\left(B^{(l)} D^{(l)}\right)^{T}\left(\left(\begin{array}{lll}
B^{(l)} & \left.D^{(l)}\right)\left(B^{(l)} D^{(l)}\right)^{T}
\end{array}\right)^{-1} \|^{2} .\right.
\end{aligned}
$$
gives

Applying norm properties on equations (71a) and (71b)

$$
\begin{aligned}
\left\|\left(\begin{array}{c}
A^{(l)} \\
C^{(l)}
\end{array}\right) \psi_{1}(k)\right\|^{2} \leq & \left\|\left(\begin{array}{c}
A^{(l)} \\
C^{(l)}
\end{array}\right) \psi_{1}(k-1)\right\|^{2}+2 \alpha_{l} \operatorname{tr}\left[\left(A^{(l)} \psi_{1}(k-1)\right)^{T}\left(-A^{(l)} \psi_{1}(k-1)-\psi_{2}(k-1) B^{(l)}\right)\right. \\
& \left.+\left(C^{(l)} \psi_{1}(k-1)\right)^{T}\left(-C^{(l)} \psi_{1}(k-1)-\psi_{2}(k-1) D^{(l)}\right)\right] \\
& +\alpha_{l}^{2}\left(\varphi_{1}\right)\left[\left\|-A^{(l)} \psi_{1}(k-1)-\psi_{2}(k-1) B^{(l)}\right\|^{2}+\left\|-C^{(l)} \psi_{1}(k-1)-\psi_{2}(k-1) D^{(l)}\right\|^{2}\right], \\
\left\|\psi_{2}(k)\left(B^{(l)} D^{(l)}\right)\right\|^{2} \leq & \left\|\psi_{2}(k-1)\left(B^{(l)} D^{(l)}\right)\right\|^{2}+2 \alpha_{l} \operatorname{tr}\left[\left(\psi_{2}(k-1) B^{(l)}\right)^{T}\left(-A^{(l)} \psi_{1}(k-1)-\psi_{2}(k-1) B^{(l)}\right)\right. \\
& \left.+\left(\psi_{2}(k-1) D^{(l)}\right)^{T}\left(-C^{(l)} \psi_{1}(k-1)-\psi_{2}(k-1) D^{(l)}\right)\right] \\
& +\alpha_{l}^{2}\left(\varphi_{2}\right)\left[\left\|-A^{(l)} \psi_{1}(k-1)-\psi_{2}(k-1) B^{(l)}\right\|^{2}+\left\|-C^{(l)} \psi_{1}(k-1)-\psi_{2}(k-1) D^{(l)}\right\|^{2}\right] .
\end{aligned}
$$

By the definition of the error in equation (66), $\|\psi(k)\|^{2} \leq\left\|\left(\begin{array}{l}A^{(l)} \\ C^{(l)}\end{array}\right) \psi_{1}(k)\right\|^{2}+\left\|\psi_{2}(k)\left(B^{(l)} D^{(l)}\right)\right\|^{2}$.
From equation (73) and by equations (72a) and (72b), the following is obtained: 


$$
\begin{aligned}
\|\psi(k)\|^{2} \leq & \left.\| \begin{array}{c}
A^{(l)} \\
C^{(l)}
\end{array}\right) \psi_{1}(k-1) \|^{2}+2 \alpha_{l} \operatorname{tr}\left[\left(A^{(l)} \psi_{1}(k-1)\right)^{T}\left(-A^{(l)} \psi_{1}(k-1)-\psi_{2}(k-1) B^{(l)}\right)\right. \\
& \left.+\left(C^{(l)} \psi_{1}(k-1)\right)^{T}\left(-C^{(l)} \psi_{1}(k-1)-\psi_{2}(k-1) D^{(l)}\right)\right] \\
& +\alpha_{l}^{2}\left(\varphi_{1}\right)\left[\left\|-A^{(l)} \psi_{1}(k-1)-\psi_{2}(k-1) B^{(l)}\right\|^{2}+\left\|-C^{(l)} \psi_{1}(k-1)-\psi_{2}(k-1) D^{(l)}\right\|^{2}\right] \\
& +\left\|\psi_{2}(k-1)\left(B^{(l)} D^{(l)}\right)\right\|^{2}+2 \alpha_{l} \operatorname{tr}\left[\left(\psi_{2}(k-1) B^{(l)}\right)^{T}\left(-A^{(l)} \psi_{1}(k-1)-\psi_{2}(k-1) B^{(l)}\right)\right. \\
& \left.+\left(\psi_{2}(k-1) D^{(l)}\right)^{T}\left(-C^{(l)} \psi_{1}(k-1)-\psi_{2}(k-1) D^{(l)}\right)\right] \\
& +\alpha_{l}^{2}\left(\varphi_{2}\right)\left[\left\|-A^{(l)} \psi_{1}(k-1)-\psi_{2}(k-1) B^{(l)}\right\|^{2}+\left\|-C^{(l)} \psi_{1}(k-1)-\psi_{2}(k-1) D^{(l)}\right\|^{2}\right], \\
\|\psi(k)\|^{2} \leq & \|\psi(k-1)\|^{2}+2 \alpha_{l} \operatorname{tr}\left[\left(A^{(l)} \psi_{1}(k-1)+\psi_{2}(k-1) B^{(l)}\right)^{T}\left(-A^{(l)} \psi_{1}(k-1)-\psi_{2}(k-1) B^{(l)}\right)\right. \\
& \left.+\left(C^{(l)} \psi_{1}(k-1)+\psi_{2}(k-1) D^{(l)}\right)^{T}\left(-C^{(l)} \psi_{1}(k-1)-\psi_{2}(k-1) D^{(l)}\right)\right] \\
& +\alpha_{l}^{2}\left(\varphi_{1}+\varphi_{2}\right)\left[\left\|-A^{(l)} \psi_{1}(k-1)-\psi_{2}(k-1) B^{(l)}\right\|^{2}+\left\|-C^{(l)} \psi_{1}(k-1)-\psi_{2}(k-1) D^{(l)}\right\|^{2}\right], \\
\|\psi(k)\|^{2} \leq & \|\psi(k-1)\|^{2}-2 \alpha_{l} \operatorname{tr}\left[\left(A^{(l)} \psi_{1}(k-1)+\psi_{2}(k-1) B^{(l)}\right)^{T}\left(A^{(l)} \psi_{1}(k-1)+\psi_{2}(k-1) B^{(l)}\right)\right. \\
& \left.+\left(C^{(l)} \psi_{1}(k-1)+\psi_{2}(k-1) D^{(l)}\right)^{T}\left(C^{(l)} \psi_{1}(k-1)-\psi_{2}(k-1) D^{(l)}\right)\right] \\
& +\alpha_{l}^{2}\left(\varphi_{1}+\varphi_{2}\right)\left[\left\|A^{(l)} \psi_{1}(k-1)+\psi_{2}(k-1) B^{(l)}\right\|^{2}+\left\|C^{(l)} \psi_{1}(k-1)+\psi_{2}(k-1) D^{(l)}\right\|^{2}\right], \\
\|\psi(k)\|^{2} \leq & \|\psi(k-1)\|^{2}-2 \alpha_{l}\left[\left\|A^{(l)} \psi_{1}(k-1)+\psi_{2}(k-1) B^{(l)}\right\|^{2}+\left\|C^{(l)} \psi_{1}(k-1)+\psi_{2}(k-1) D^{(l)}\right\|^{2}\right] \\
& +\alpha_{l}^{2}\left(\varphi_{1}+\varphi_{2}\right)\left[\left\|A^{(l)} \psi_{1}(k-1)+\psi_{2}(k-1) B^{(l)}\right\|^{2}+\left\|C^{(l)} \psi_{1}(k-1)+\psi_{2}(k-1) D^{(l)}\right\|^{2}\right] .
\end{aligned}
$$

By equation (62), the following can be obtained:

$$
\begin{aligned}
\|\psi(k)\|^{2} \leq & \|\psi(k-1)\|^{2}-\alpha_{l}\left(2-\alpha_{l}\left(\varphi_{1}+\varphi_{2}\right)\left[\left\|A^{(l)} \psi_{1}(k-1)+\psi_{2}(k-1) B^{(l)}\right\|^{2}\right.\right. \\
& \left.+\left\|C^{(l)} \psi_{1}(k-1)+\psi_{2}(k-1) D^{(l)}\right\|^{2}\right] .
\end{aligned}
$$

At $k=1,\|\psi(1)\|^{2} \leq\|\psi(0)\|^{2}-\alpha_{l}\left(2-\alpha_{l}\left(\varphi_{1}+\varphi_{2}\right)\right)\left[\| A^{(l)}\right.$ $\left.\psi_{1}(0)+\psi_{2}(0) B^{(l)}\left\|^{2}+\right\| C^{(l)} \psi_{1}(0)+\psi_{2}(0) D^{(l)} \|^{2}\right]$.

At $k=2,\|\psi(2)\|^{2} \leq\|\psi(1)\|^{2}-\alpha_{l}\left(2-\alpha_{l}\left(\varphi_{1}+\varphi_{2}\right)\right)\left[\| A^{(l)}\right.$ $\left.\psi_{1}(1)+\psi_{2}(1) B^{(l)}\left\|^{2}+\right\| C^{(l)} \psi_{1}(1)+\psi_{2}(1) D^{(l)} \|^{2}\right]$.

At $k=3,\|\psi(3)\|^{2} \leq\|\psi(2)\|^{2}-\alpha_{l}\left(2-\alpha_{l}\left(\varphi_{1}+\varphi_{2}\right)\right)\left[\| A^{(l)}\right.$ $\left.\psi_{1}(2)+\psi_{2}(2) B^{(l)}\left\|^{2}+\right\| C^{(l)} \psi_{1}(2)+\psi_{2}(2) D^{(l)} \|^{2}\right]$.
At $k=n-1,\|\psi(n-1)\|^{2} \leq\|\psi(n-2)\|^{2}-\alpha_{l}\left(2-\alpha_{l}\left(\varphi_{1}+\right.\right.$ $\left.\left.\varphi_{2}\right)\right) \quad\left[\left\|A^{(l)} \psi_{1}(n-2)+\psi_{2}(n-2) B^{(l)}\right\|^{2}+\| C^{(l)} \psi_{1}(n-2)+\right.$ $\left.\psi_{2}(n-2) D^{(l)} \|^{2}\right]$.

At $\quad k=n, \quad\|\psi(n)\|^{2} \leq\|\psi(n-1)\|^{2} \alpha_{l}\left(2-\alpha_{l}\left(\varphi_{1}+\varphi_{2}\right)\right)$ $\left[\left\|A^{(l)} \psi_{1}(n-1)+\psi_{2}(n-1) B^{(l)}\right\|^{2}+\| C^{(l)} \psi_{1}(n-1)+\psi_{2}\right.$ $\left.(n-1) D^{(l)} \|^{2}\right]$.

Therefore, the following is obtained:

$$
\begin{aligned}
\|\psi(k)\|^{2} \leq & \|\psi(k-1)\|^{2}-\alpha_{l}\left(\left(2-\alpha_{l}\left(\varphi_{1}+\varphi_{2}\right)\left[\left\|a_{i j}^{(l)} \psi_{1}(n-1)+\psi_{2}(n-1) b_{i j}^{(l)}\right\|^{2}\right.\right.\right. \\
& \left.+\left\|c_{i j}^{(l)} \psi_{1}(k-1)+\psi_{2}(k-1) d_{i j}^{(l)}\right\|^{2}\right]
\end{aligned}
$$

If the convergence factor $\alpha$ is chosen to satisfy equation (41b) and $k \longrightarrow \infty$, then

$$
\sum_{k=1}^{\infty}\left(\left\|A^{(l)} \psi_{1}(k)+\psi_{2}(k) B^{(l)}\right\|^{2}+\left\|C^{(l)} \psi_{1}(k)+\psi_{2}(k) D^{(l)}\right\|^{2}\right)<\infty .
$$

Therefore,

$$
\begin{aligned}
& \lim _{k \longrightarrow \infty}\left(A^{(l)} \psi_{1}(k)+\psi_{2}(k) B^{(l)}\right)=0, \\
& \lim _{k \longrightarrow \infty}\left(C^{(l)} \psi_{1}(k)+\psi_{2}(k) D^{(l)}\right)=0 .
\end{aligned}
$$


Input that $\widetilde{A}, \widetilde{B}, \widetilde{C}, \widetilde{D}, \widetilde{E}, \widetilde{F}$ \# Split each matrix into four matrices (e.g., $A^{(1)}, A^{(2)}, A^{(3)}, A^{(4)}$ )

for $l=1,2,3,4$

Choose $\alpha_{l}, \varepsilon, \hat{x}_{l}(0)=0, \hat{y}_{l}(0)=0$ \# 0 is the Zero matrix with the same dimension as $X^{(l)}(k)$ and $Y^{(l)}(k)$.

While $k=0,1,2, \ldots, n$ do

$$
\begin{aligned}
& \left\{\begin{array}{l}
\hat{x}_{l}(k)=\hat{x}_{l}(k-1)+\alpha_{l}\left(\left(\gamma_{l}\right)^{T} \gamma_{l}\right)^{-1}\left(\gamma_{l}\right)^{T}\left(\begin{array}{l}
r_{l}(k-1) \\
s_{l}(k-1)
\end{array}\right) \\
\hat{y}_{l}(k)=\hat{y}_{l}(k-1)+\alpha_{l} \cdot\left(r_{l}(k-1) s_{l}(k-1)\right)\left(\beta_{l}\right)^{T}\left(\left(\beta_{l}\right)^{T} \beta_{l}\right)^{-1}
\end{array}\right. \\
& r_{l}(k-1)=E^{(l)}-A^{(l)} \hat{x}_{l}(k-1)-\hat{y}_{l}(k-1) B^{(l)} \\
& s_{l}(k-1)=F^{(l)}-C^{(l)} \hat{x}_{l}(k-1)-\hat{y}_{l}(k-1) D^{(l)} \\
& \gamma_{l}=\left(\begin{array}{c}
a_{i j}^{(l)} \\
c_{i j}^{(l)}
\end{array}\right), \beta_{l}=\left(\begin{array}{ll}
b_{i j}^{(l)} & d_{i j}^{(l)}
\end{array}\right) \\
& \delta_{l}(k)=\sqrt{\left(\left(\left\|\hat{x}_{l}(k)-\hat{x}_{l}(k-1)\right\|^{2}+\left\|\hat{y}_{l}(k)-\hat{y}_{l}(k-1)\right\|^{2}\right) /\left(\left\|\hat{x}_{l}(k)\right\|^{2}+\left\|\hat{y}_{l}(k)\right\|^{2}\right)\right)} \\
& \text { If } \delta_{l}(k)<\varepsilon \text { then } \\
& \text { print }\left(x_{l}(k), \hat{y}_{l}(k)\right) \text {; } \\
& \text { print ("number of iterations }=", k) \text {. } \\
& \left\{\begin{array}{l}
\hat{x}_{l}(k)=\hat{x}_{l}(k-1)+\alpha_{l} \cdot\left(\gamma_{l}\right)^{T}\left(\begin{array}{l}
r_{l}(k-1) \\
s_{l}(k-1)
\end{array}\right) \\
\hat{y}_{l}(k)=\hat{y}_{l}(k-1)+\alpha_{l} \cdot\left(r_{l}(k-1) s_{l}(k-1)\right)\left(\beta_{l}\right)^{T}
\end{array},\right. \\
& \text { update } k \text {. } \\
& k=k+1 \\
& \text { end } \\
& \text { print }\left(\hat{x}_{l}(k), \hat{y}_{l}(k)\right) \text {; } \\
& \text { print (" number of iterations }=", k) \text {. }
\end{aligned}
$$

else

end

Algorithm 3: FLSI algorithm for CTrFFSME

Since $A^{(l)}>0, B^{(l)}>0, C^{(l)}>0$, and $D^{(l)}>0$, then

$$
\begin{aligned}
& \lim _{k \longrightarrow \infty} \psi_{1}(k)=0, \\
& \lim _{k \longrightarrow \infty} \psi_{2}(k)=0 .
\end{aligned}
$$

By equations (46a) and (46b), the following is obtained:

$$
\begin{aligned}
& \lim _{k \rightarrow \infty}\left(X^{(l)}-\hat{x}^{(l)}(k)\right)=0, \\
& \lim _{k \rightarrow \infty}\left(Y^{(l)}-\hat{y}^{(l)}(k)\right)=0 .
\end{aligned}
$$

Consequently, if $k \longrightarrow \infty$, then $X^{(l)}=\hat{x}^{(l)}(k)$ and $Y^{(l)}=\hat{y}^{(k)}(k)$

Therefore, if the system of CSME in equation (24) has a unique positive solution $\left(X^{(l)}, Y^{(l)}\right)$, then the iterative solution $\left(\hat{x}^{(l)}(k), \hat{y}^{(l)}(k)\right)$ in equation (60) converges to
$\left(X^{(l)}, Y^{(l)}\right)$ for any initial values $\hat{x}^{(l)}(0), \hat{y}^{(l)}(0)$ for $1 \leq l \leq 4$ (i.e., if $k \longrightarrow \infty$, then $X^{(l)}=\hat{x}^{(l)}(k)$ and $Y^{(l)}=\hat{y}^{(l)}(k)$ ).

Algorithm 3 summarizes the FLSI, respectively. This algorithm can be implemented by any mathematical software for solving the CTrFFSME in equation (1).

In Section 3.4, the three proposed methods for solving the CTrFFSME in equation (1) are applied to different fuzzy systems.

3.4. Applications of the Proposed Methods to Other Fuzzy Systems and Fuzzy Numbers. The proposed methods can solve different positive fuzzy systems with triangular and trapezoidal fuzzy numbers. It can be directly applied to the following.

The fully fuzzy matrix equation is as follows: 


$$
\widetilde{A} \widetilde{X}=\widetilde{E} .
$$

The fully fuzzy Sylvester matrix equation is as follows:

$$
\widetilde{A} \tilde{X}+\tilde{X} \widetilde{D}=\widetilde{E} .
$$

The fully fuzzy continuous-time Lyapunov matrix equation is as follows:

$$
\widetilde{A} \widetilde{X}+\widetilde{X} \widetilde{A}^{T}=\widetilde{E}
$$

The next section illustrates the three proposed methods by solving two examples sized $2 \times 2$ and $100 \times 100$, followed by the solutions verification.

\section{Numerical Examples}

To illustrate the accuracy and effectiveness of the proposed methods for solving the CTrFFSME in equation (1), we consider various sizes of CTrFFSME, namely, small $(2 \times 2)$ and large $(100 \times 100)$. Analytical solutions are found by Algorithm 1 for FMVM. Then, we compare the performance of Algorithm 2 for FGI and FLSI for approximating that solution by calculating the number of iterations $(k)$, convergence factor $(\alpha)$, error bound $(\varepsilon)$, convergence rate, CPU time, real-time, and memory usage. In addition to the graphical representation of the relative error $\delta^{l}(k)$, when $k$ increases, in Example 1, the proposed methods are applied to small CTrFFSME $(2 \times 2)$.

Example 1. Solve the following $2 \times 2$ CTrFFSME:

$$
\left\{\begin{array}{l}
\widetilde{A} \widetilde{X}+\widetilde{Y} \widetilde{B}=\widetilde{E} \\
\widetilde{C} \widetilde{X}+\widetilde{Y} \widetilde{D}=\widetilde{F}
\end{array}\right.
$$

Given

$$
\begin{aligned}
\widetilde{A} & =\left(\begin{array}{ll}
(2,3,5,7) & (1,2,4,6) \\
(1,2,3,5) & (2,4,6,9)
\end{array}\right), \\
\widetilde{B} & =\left(\begin{array}{ll}
(2,4,5,8) & (3,6,8,10) \\
(3,5,7,9) & (1,2,4,6)
\end{array}\right), \\
\widetilde{E} & =\left(\begin{array}{ll}
(17,48,110,252) & (17,48,114,240) \\
(21,63,130,293) & (20,66,142,289)
\end{array}\right), \\
\widetilde{C} & =\left(\begin{array}{ll}
(2,4,5,7) & (5,7,9,11) \\
(5,6,7,8) & (2,3,4,6)
\end{array}\right), \\
\widetilde{D} & =\left(\begin{array}{ll}
(1,3,4,6) & (3,4,6,8) \\
(2,3,5,7) & (4,5,7,9)
\end{array}\right), \\
\widetilde{F} & =\left(\begin{array}{ll}
(22,59,121,267) & (36,85,161,305) \\
(32,66,128,258) & (41,83,159,292)
\end{array}\right) .
\end{aligned}
$$

Solution. The solution to the given positive CTrFFSME is obtained by the proposed methods as follows.

4.1. Fuzzy Matrix Vectorization Method (FMVM). By decomposing the given positive CTrFFSME and applying Algorithm 1 for FMVM, the analytical solution is obtained as follows:

Step 1. Convert the given CTrFFSME to four systems of CSME using Theorem 3.

Step 2. Apply Vec-operator and Kronecker product on the systems obtained in Step 1.

Step 3. Convert the obtained systems in Step 2 to the linear system $P Q=U$

where 


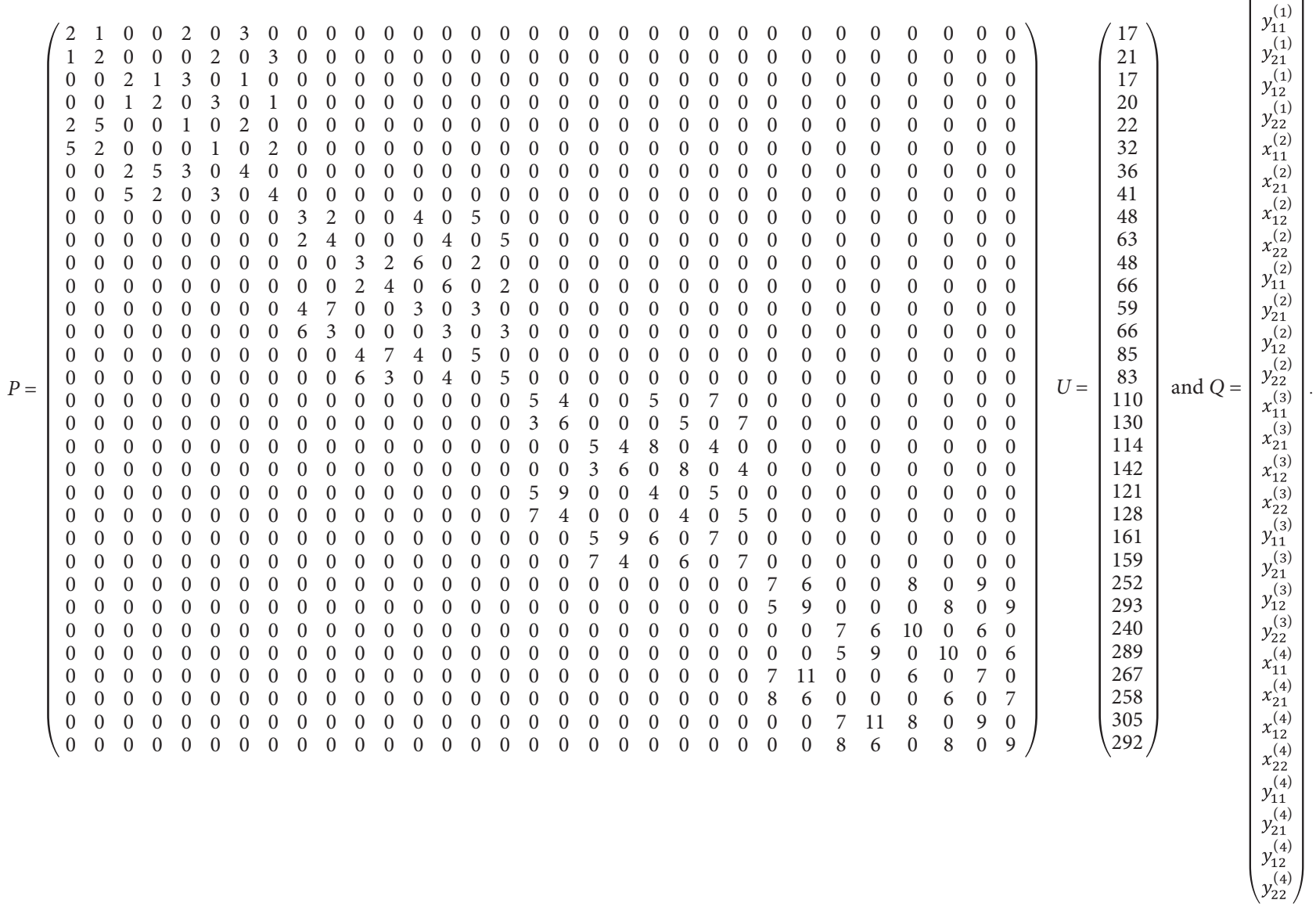

(86)

Step 4. Multiplying both sides of the system obtained in Step 3 by $P^{-1}$ and solving for $Q$, we get 
TABLe 1: Comparison between FFMVM, FGI, and FLSI for Example 1.

\begin{tabular}{|c|c|c|c|c|c|}
\hline & Method & Analytical solution-approximated solution & $\alpha_{l}$ & $\varepsilon$ & $k$ \\
\hline \multirow{3}{*}{$\hat{x}^{(1)}$} & FMVM & $\left(\begin{array}{ll}4 & 3 \\
2 & 4\end{array}\right)$ & NA & 0 & NA \\
\hline & FGI & $\left(\begin{array}{ll}4.0003146781142283762 & 3.0008763877876229244 \\
1.9996620682548063892 & 3.9991059886583548709\end{array}\right)$ & 0.01515 & $10^{-5}$ & 236 \\
\hline & FLSI & $\left(\begin{array}{ll}3.9994905651474607941 & 2.9991905860792312089 \\
1.9994905651470906697 & 3.9991905860781175687\end{array}\right)$ & 0.5 & $10^{-5}$ & 213 \\
\hline \multirow{3}{*}{$\hat{y}^{(1)}$} & FMVM & $\left(\begin{array}{ll}2 & 1 \\
2 & 3\end{array}\right)$ & NA & 0 & NA \\
\hline & FGI & $\left(\begin{array}{ll}1.9988524913049122353 & 1.0013283860149024908 \\
2.0010864923386014879 & 2.9987621291364226938\end{array}\right)$ & 0.01515 & $10^{-5}$ & 236 \\
\hline & FLSI & $\left(\begin{array}{ll}2.0005343495876688624 & 1.0009007873088771599 \\
2.0005343495884565574 & 3.0009007873076832875\end{array}\right)$ & 0.5 & $10^{-5}$ & 213 \\
\hline
\end{tabular}

\begin{tabular}{|c|c|c|c|c|c|}
\hline \multirow{3}{*}{$\hat{x}^{(2)}$} & FMVM & $\left(\begin{array}{ll}5 & 4 \\
3 & 6\end{array}\right)$ & NA & 0 & NA \\
\hline & FGI & $\left(\begin{array}{cc}4.999864079584862688 & 4.0030110768713679898 \\
3.0003389970085296313 & 5.997280209950071278\end{array}\right)$ & 0.006711 & $10^{-5}$ & 333 \\
\hline & FLSI & $\left(\begin{array}{ll}4.9988761522873104256 & 3.9985435355183413819 \\
2.9990633422322140346 & 5.9990330988581697249\end{array}\right)$ & 0.5 & $10^{-5}$ & 184 \\
\hline \multirow{3}{*}{$\hat{y}^{(2)}$} & FMVM & $\left(\begin{array}{ll}3 & 3 \\
4 & 5\end{array}\right)$ & NA & 0 & NA \\
\hline & FGI & $\left(\begin{array}{ll}2.9983853131430880787 & 3.0019716289620118953 \\
4.0023361161663756989 & 4.9968909026675074104\end{array}\right)$ & 0.006711 & $10^{-5}$ & 333 \\
\hline & FLSI & $\left(\begin{array}{cc}3.0006934360840940105 & 3.0016140300903587928 \\
4.0008105860628660745 & 5.001423238975299027\end{array}\right)$ & 0.5 & $10^{-5}$ & 184 \\
\hline \multirow{3}{*}{$\hat{x}^{(3)}$} & FMVM & $\left(\begin{array}{ll}7 & 6 \\
5 & 8\end{array}\right)$ & NA & 0 & NA \\
\hline & FGI & $\left(\begin{array}{ll}6.9966176458091605751 & 5.9986527914727380584 \\
4.9980891197185313267 & 7.9959255849905723918\end{array}\right)$ & 0.003436 & $10^{-5}$ & 269 \\
\hline & FLSI & $\left(\begin{array}{ll}6.9949554419054769516 & 5.9938085953915972691 \\
4.9961576166547481863 & 7.9958332089102526017\end{array}\right)$ & 0.5 & $10^{-5}$ & 367 \\
\hline \multirow{3}{*}{$\hat{y}^{(3)}$} & FMVM & $\left(\begin{array}{ll}4 & 5 \\
6 & 7\end{array}\right)$ & NA & 0 & NA \\
\hline & FGI & $\left(\begin{array}{cc}3.9992591622654458963 & 5.006245036314479103 \\
6.0032068410122893031 & 7.0014106935371849297\end{array}\right)$ & 0.003436 & $10^{-5}$ & 269 \\
\hline & FLSI & $\left(\begin{array}{ll}4.0034261490303281354 & 5.0060523578143844872 \\
6.0032467233815930657 & 7.0052034740382072243\end{array}\right)$ & 0.5 & $10^{-5}$ & 367 \\
\hline \multirow{3}{*}{$\hat{x}^{(4)}$} & FMVM & $\left(\begin{array}{cc}10 & 8 \\
9 & 11\end{array}\right)$ & NA & 0 & NA \\
\hline & FGI & $\left(\begin{array}{cc}9.9965893617203098369 & 8.0200665293211628219 \\
8.99879605836685188 & 10.978609176105587048\end{array}\right)$ & 0.001831 & $10^{-5}$ & 551 \\
\hline & FLSI & $\left(\begin{array}{ll}9.9826328737657879555 & 7.9801395018407377488 \\
8.9911911526917516843 & 10.991796352652112364\end{array}\right)$ & 0.5 & $10^{-5}$ & 502 \\
\hline \multirow{3}{*}{$\hat{y}^{(4)}$} & FMVM & $\left(\begin{array}{cc}7 & 8 \\
9 & 10\end{array}\right)$ & NA & 0 & NA \\
\hline & FGI & $\left(\begin{array}{ll}6.9831466534571057456 & 8.0224337286592531978 \\
9.0229413143636603062 & 9.9800757765377075115\end{array}\right)$ & 0.001831 & $10^{-5}$ & 551 \\
\hline & FLSI & $\left(\begin{array}{cc}7.0083279806078923944 & 8.017488243908286638 \\
9.0079819495371672092 & 10.015609497910344443\end{array}\right)$ & 0.5 & $10^{-5}$ & 502 \\
\hline
\end{tabular}


TABLe 2: Computational time and memory usage for FGI and FLSI for Example 1.

\begin{tabular}{|c|c|c|c|c|c|}
\hline & Method & $k$ & CPU time $(\mathrm{ms})$ & Real-time (ms) & Memory usage (MB) \\
\hline \multirow{2}{*}{$\hat{\Lambda}^{(1)}$} & FGI & 236 & 6.09 & 6.15 & 1.05 \\
\hline & FLSI & 213 & 8.15 & 9.89 & 1.32 \\
\hline \multirow{2}{*}{$\hat{Y}^{(1)}$} & FGI & 236 & 6.29 & 6.15 & 1.05 \\
\hline & FLSI & 213 & 7.63 & 7.73 & 1.30 \\
\hline \multirow{2}{*}{$\hat{X}^{(2)}$} & FGI & 333 & 6.24 & 6.17 & 1.05 \\
\hline & FLSI & 184 & 7.39 & 7.38 & 1.32 \\
\hline \multirow{2}{*}{$\hat{\stackrel{Y}{Y}}^{(2)}$} & FGI & 333 & 6.15 & 6.19 & 1.05 \\
\hline & FLSI & 184 & 7.13 & 7.30 & 1.30 \\
\hline \multirow{2}{*}{$\hat{X}^{(3)}$} & FGI & 269 & 6.16 & 6.09 & 1.05 \\
\hline & FLSI & 367 & 7.71 & 7.76 & 1.32 \\
\hline \multirow{2}{*}{$\hat{\mathrm{Y}}^{(3)}$} & FGI & 269 & 6.16 & 6.06 & 1.05 \\
\hline & FLSI & 367 & 8.00 & 7.99 & 1.30 \\
\hline \multirow{2}{*}{$\hat{\wedge}^{(4)}$} & FGI & 551 & 5.98 & 5.99 & 1.05 \\
\hline & FLSI & 502 & 7.91 & 7.86 & 1.32 \\
\hline \multirow{2}{*}{$\hat{Y}^{(4)}$} & FGI & 551 & 6.10 & 5.99 & 1.05 \\
\hline & FLSI & 502 & 7.66 & 7.71 & 1.30 \\
\hline
\end{tabular}

$$
Q=\left(\begin{array}{c}
4 \\
2 \\
3 \\
4 \\
2 \\
2 \\
1 \\
3 \\
5 \\
3 \\
4 \\
6 \\
3 \\
4 \\
3 \\
5 \\
7 \\
5 \\
6 \\
8 \\
4 \\
6 \\
5 \\
7 \\
10 \\
9 \\
8 \\
11 \\
7 \\
9 \\
8 \\
10
\end{array}\right)
$$

Step 5. By Definitions 9 and 10, the obtained solution in Step 4 can be written as follows:

$$
\left\{\begin{array}{c}
\widetilde{X}=\left(\begin{array}{cc}
(4,5,7,10) & (3,4,6,8) \\
(2,3,5,9) & (4,6,8,11)
\end{array}\right), \\
\widetilde{Y}=\left(\begin{array}{ll}
(2,3,4,7) & (1,3,5,8) \\
(2,4,6,9) & (3,5,7,10)
\end{array}\right) .
\end{array}\right.
$$

This positive fuzzy solution is approximated using Algorithms 2 and 3 as follows.

4.2. Fuzzy Gradient Iterative (FGI) Method and Fuzzy LeastSquare Iterative (FLSI) Method. Algorithms 2 and 3 for FGI and FLSI are applied to compute the approximated fuzzy solutions $\hat{x}^{(k)}$ and $\hat{y}^{(k)}$ for the given CTrFFSME using the following initial value for $1 \leq l \leq 4$, $\hat{x}^{(l)}=\left(\begin{array}{ll}0 & 0 \\ 0 & 0\end{array}\right)$ and $\hat{y}^{(l)}=\left(\begin{array}{ll}0 & 0 \\ 0 & 0\end{array}\right)$. The approximated solutions $\hat{X}$ and $\hat{Y}$ are shown in Table 1 with the convergence factor $\left(\alpha_{l}\right)$, error bound $(\varepsilon)$, and the total number of iteration $(k)$.

Table 2 shows the computational time and memory usage for FGI and FLSI.

Figure 2 shows the change in the relative error $\delta^{l}(k)$ when $k$ increases up to $k=20$.

From Tables 1 and 2 and Figure 2, the relative error $\delta^{l}(k)$ is becoming smaller as $k$ increases. This indicates that the 


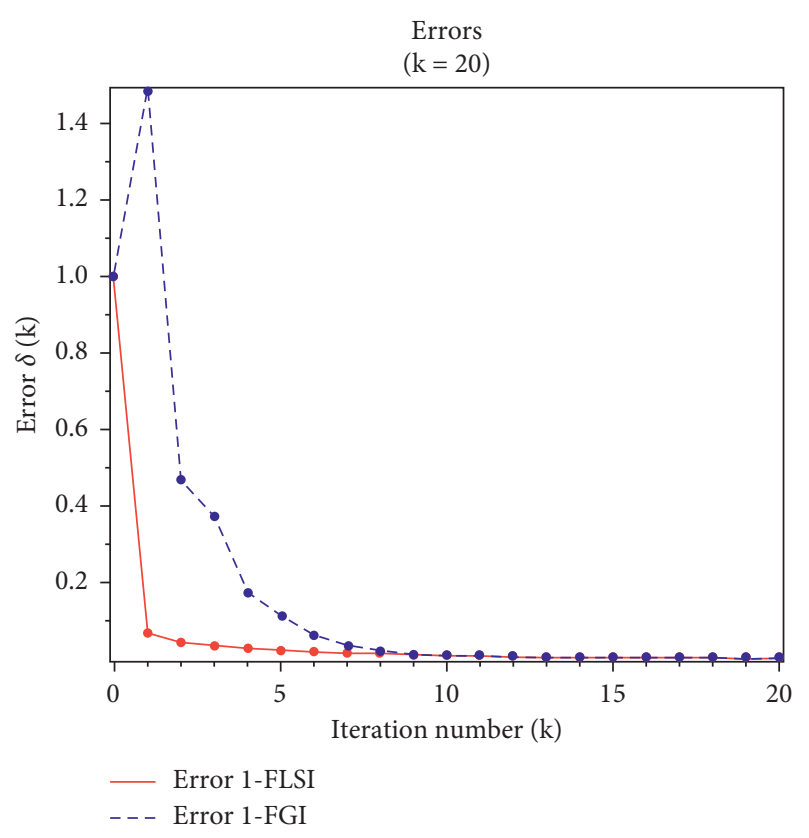

(a)

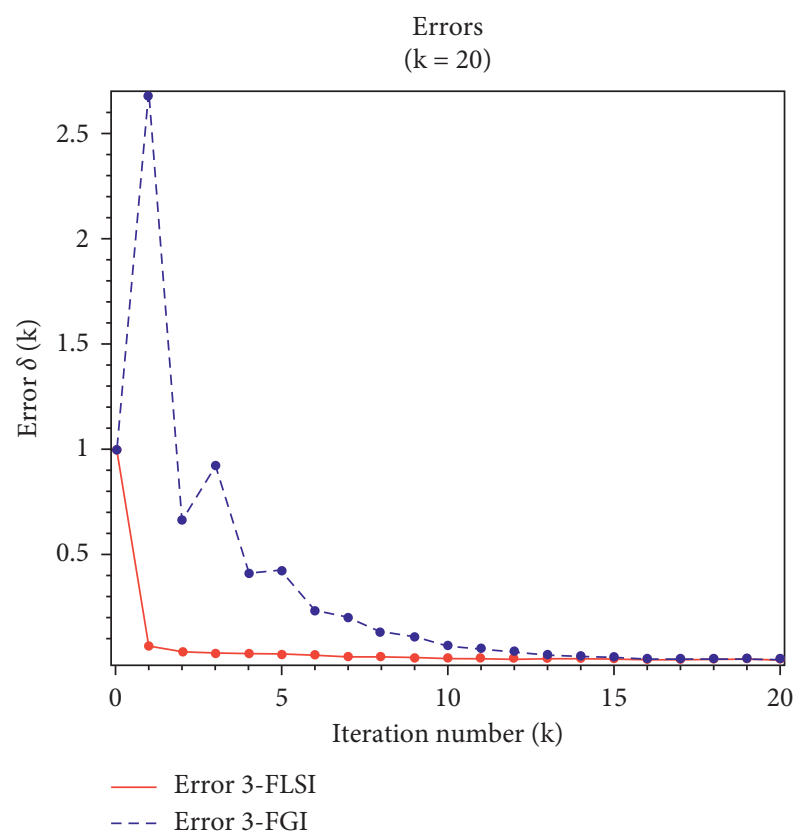

(c)

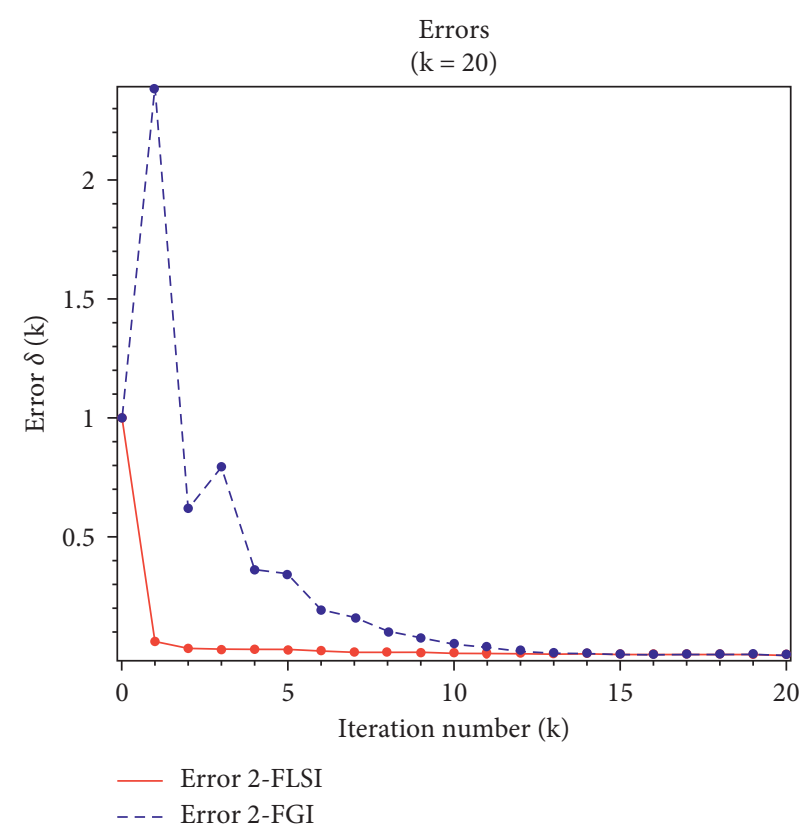

(b)

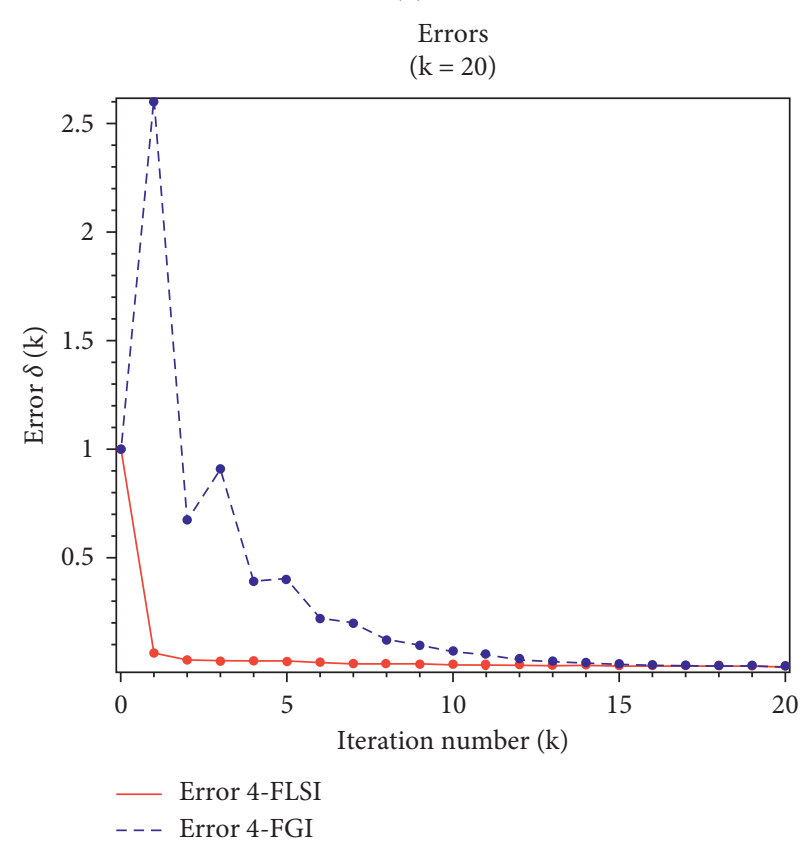

(d)

FIgURE 2: Comparison between $\delta^{l}(k)$ of FGI and FLSI for the first 20 iterations for Example 1.

proposed methods are effective and convergent for the given CTrFFSME. This indicates that the proposed algorithms are effective and convergent for the given positive CTrFFSME.
4.3. Verification of the Solution. To verify the obtained fuzzy solution, we first multiply $\widetilde{A} \widetilde{X}$ as follows:

$$
\begin{aligned}
& \widetilde{A} \widetilde{X}=\left(\begin{array}{ll}
(2,3,5,7) & (1,2,4,6) \\
(1,2,3,5) & (2,4,6,9)
\end{array}\right)\left(\begin{array}{cc}
(4,5,7,10) & (3,4,6,8) \\
(2,3,5,9) & (4,6,8,11)
\end{array}\right)=\left(\begin{array}{cc}
(10,21,55,124) & (10,24,62,122) \\
(8,22,51,131) & (11,32,66,139)
\end{array}\right), \\
& \widetilde{Y} \widetilde{B}=\left(\begin{array}{ll}
(2,3,5,7) & (1,3,5,8) \\
(2,4,6,9) & (3,5,7,10)
\end{array}\right)\left(\begin{array}{cc}
(2,4,5,8) & (3,6,8,10) \\
(3,5,7,9) & (1,2,4,6)
\end{array}\right)=\left(\begin{array}{cc}
(7,27,55,128) & (7,24,52,118) \\
(13,41,79,162) & (9,34,76,150)
\end{array}\right) .
\end{aligned}
$$


We also multiply $\widetilde{C} \widetilde{X}$ as follows:

$$
\begin{aligned}
& \widetilde{C} \widetilde{X}=\left(\begin{array}{ll}
(1,3,4,6) & (3,4,6,8) \\
(2,3,5,7) & (4,5,7,9)
\end{array}\right)\left(\begin{array}{cc}
(4,5,7,10) & (3,4,6,8) \\
(2,3,5,9) & (4,6,8,11)
\end{array}\right)=\left(\begin{array}{ll}
(18,41,80,169) & (26,58,102,177) \\
(24,39,69,134) & (23,42,74,130)
\end{array}\right), \\
& \widetilde{Y} \widetilde{D}=\left(\begin{array}{cc}
(2,3,5,7) & (1,3,5,8) \\
(2,4,6,9) & (3,5,7,10)
\end{array}\right)\left(\begin{array}{cc}
(2,4,5,7) & (5,7,9,11) \\
(5,6,7,8) & (2,3,4,6)
\end{array}\right)=\left(\begin{array}{cc}
(4,18,41,98) & (10,27,59,128) \\
(8,27,59,124) & (18,41,85,162)
\end{array}\right) .
\end{aligned}
$$

Therefore,

$$
\begin{aligned}
& \widetilde{A} \widetilde{X}+\widetilde{Y} \widetilde{B}=\left(\begin{array}{ll}
(17,48,110,252) & (17,48,114,240) \\
(21,63,130,293) & (20,66,142,289)
\end{array}\right)=\widetilde{E}, \\
& \widetilde{C} \widetilde{X}+\widetilde{Y} \widetilde{D}=\left(\begin{array}{ll}
(22,59,121,267) & (36,85,161,305) \\
(32,66,128,258) & (41,83,159,292)
\end{array}\right)=\widetilde{F}
\end{aligned}
$$

The obtained positive fuzzy solution satisfies the given CTrFFSME, and it is feasible (strong fuzzy solution).
In Example 2, we tested the proposed method on $100 \times$ 100 CTrFFSME, and we obtained the following results using Maple 2019.

Example 2. Solve the following $100 \times 100$ CTrFFSME:

$$
\left\{\begin{array}{l}
\widetilde{A} \widetilde{X}+\widetilde{Y} \widetilde{B}=\widetilde{E}, \\
\widetilde{C} \widetilde{X}+\widetilde{Y} \widetilde{D}=\widetilde{F}
\end{array}\right.
$$

Given

$$
\begin{aligned}
& A^{(1)}=\text { LinearAlgebra: }- \text { RandomMatrix }(100,100 \text {, generator }=1 \cdots 2) \text {, } \\
& B^{(1)}=\text { LinearAlgebra: - RandomMatrix }(100,100 \text {, generator }=1 \cdots 2) \text {, } \\
& C^{(1)}=\text { LinearAlgebra: }- \text { RandomMatrix }(100,100 \text {, generator }=1 \cdots 2) \text {, } \\
& D^{(1)}=\text { LinearAlgebra: }- \text { RandomMatrix }(100,100 \text {, generator }=1 \cdots 2) \text {, } \\
& E^{(1)}=\text { LinearAlgebra: }- \text { RandomMatrix }\left(100,100 \text {, generator }=7 \times 10^{2} \cdots 1.5 \times 10^{3}\right) \text {, } \\
& F^{(1)}=\text { LinearAlgebra: }- \text { RandomMatrix }\left(100,100 \text {, generator }=7 \times 10^{2} \cdots 1.5 \times 10^{3}\right) \text {, } \\
& A^{(2)}=\text { LinearAlgebra: - RandomMatrix }(100,100 \text {, generator }=3 \cdots 4) \text {, } \\
& B^{(2)}=\text { LinearAlgebra: - RandomMatrix }(100,100 \text {, generator }=3 \cdots 4) \text {, } \\
& C^{(2)}=\text { LinearAlgebra: - RandomMatrix }(100,100 \text {, generator }=3 \cdots 4) \text {, } \\
& D^{(2)}=\text { LinearAlgebra: - RandomMatrix }(100,100 \text {, generator }=3 \cdots 4) \text {, } \\
& E^{(2)}=\text { LinearAlgebra: }- \text { RandomMatrix }\left(100,100 \text {, generator }=4 \times 10^{4} \cdots 6 \times 10^{4}\right) \text {, } \\
& F^{(2)}=\text { LinearAlgebra: }- \text { RandomMatrix }\left(100,100 \text {, generator }=4 \times 10^{4} \cdots 6 \times 10^{4}\right) \text {, } \\
& A^{(3)}=\text { LinearAlgebra: - RandomMatrix }(100,100 \text {, generator }=5 \cdots 6) \text {, } \\
& B^{(3)}=\text { LinearAlgebra: }- \text { RandomMatrix }(100,100 \text {, generator }=5 \cdots 6) \text {, } \\
& C^{(3)}=\text { LinearAlgebra: }- \text { RandomMatrix }(100,100 \text {, generator }=5 \cdots 6) \text {, } \\
& D^{(3)}=\text { LinearAlgebra: - RandomMatrix }(100,100 \text {, generator }=5 \cdots 6) \text {, } \\
& E^{(3)}=\text { LinearAlgebra: }- \text { RandomMatrix }\left(100,100 \text {, generator }=1.1 \times 10^{4} \cdots 1.3 \times 10^{4}\right) \text {, } \\
& F^{(3)}=\text { LinearAlgebra: }- \text { RandomMatrix }\left(100,100 \text {, generator }=1.1 \times 10^{4} \cdots 1.3 \times 10^{4}\right) \text {, } \\
& A^{(4)}=\text { LinearAlgebra: - RandomMatrix }(100,100 \text {, generator }=7 \cdots 8) \text {, } \\
& B^{(4)}=\text { LinearAlgebra: }- \text { RandomMatrix }(100,100 \text {, generator }=7 \cdots 8) \text {, } \\
& C^{(4)}=\text { LinearAlgebra: - RandomMatrix }(100,100 \text {, generator }=7 \cdots 8) \text {, } \\
& D^{(4)}=\text { LinearAlgebra: - RandomMatrix }(100,100 \text {, generator }=7 \cdots 8) \text {, } \\
& E^{(4)}=\text { LinearAlgebra: - RandomMatrix }\left(100,100 \text {, generator }=2.2 \times 10^{5} \cdots 3 \times 10^{5}\right) \text {, } \\
& F^{(4)}=\text { LinearAlgebra: }- \text { RandomMatrix }\left(100,100 \text {, generator }=2.2 \times 10^{5} \cdots 3 \times 10^{5}\right) \text {. }
\end{aligned}
$$


TABLe 3: Comparison between FGI and FLSI for Example 2.

\begin{tabular}{|c|c|c|c|c|c|c|}
\hline & Method & $k$ & $\alpha_{l}$ & CPU time (s) & Real-time (s) & Memory usage (GB) \\
\hline \multirow{2}{*}{$\hat{x}^{(1)}$} & FGI & 10 & $2 \times 10^{-7}$ & 7.58 & 6.17 & 1.40 \\
\hline & FLSI & 10 & 0.999 & 22.51 & 19.06 & 2.52 \\
\hline \multirow{2}{*}{$\hat{y}^{(1)}$} & FGI & 10 & $2 \times 10^{-7}$ & 7.73 & 6.29 & 1.35 \\
\hline & FLSI & 10 & 0.999 & 22.34 & 19.38 & 2.45 \\
\hline \multirow{2}{*}{$\hat{x}^{(2)}$} & FGI & 10 & $2 \times 10^{-7}$ & 7.83 & 6.4 & 1.43 \\
\hline & FLSI & 10 & 0.999 & 25.69 & 22.54 & 2.78 \\
\hline \multirow{2}{*}{$\hat{y}^{(2)}$} & FGI & 10 & $2 \times 10^{-7}$ & 8.28 & 6.73 & 1.43 \\
\hline & FLSI & 10 & 0.999 & 25.75 & 22.62 & 2.78 \\
\hline \multirow{2}{*}{$\hat{x}^{(3)}$} & FGI & 10 & $2 \times 10^{-7}$ & 7.72 & 6.29 & 1.45 \\
\hline & FLSI & 10 & 0.999 & 27.32 & 24.24 & 2.91 \\
\hline \multirow{2}{*}{$\hat{y}^{(3)}$} & FGI & 10 & $2 \times 10^{-7}$ & 8.26 & 6.71 & 1.45 \\
\hline & FLSI & 10 & 0.999 & 27.58 & 24.36 & 3.02 \\
\hline \multirow{2}{*}{$\hat{x}^{(4)}$} & FGI & 10 & $2 \times 10^{-7}$ & 8.85 & 7.11 & 1.60 \\
\hline & FLSI & 10 & 0.999 & 28.84 & 25.47 & 3.09 \\
\hline \multirow{2}{*}{$\hat{y}^{(4)}$} & FGI & 10 & $2 \times 10^{-7}$ & 9.10 & 7.37 & 1.60 \\
\hline & FLSI & 10 & 0.999 & 28.80 & 25.44 & 3.09 \\
\hline
\end{tabular}

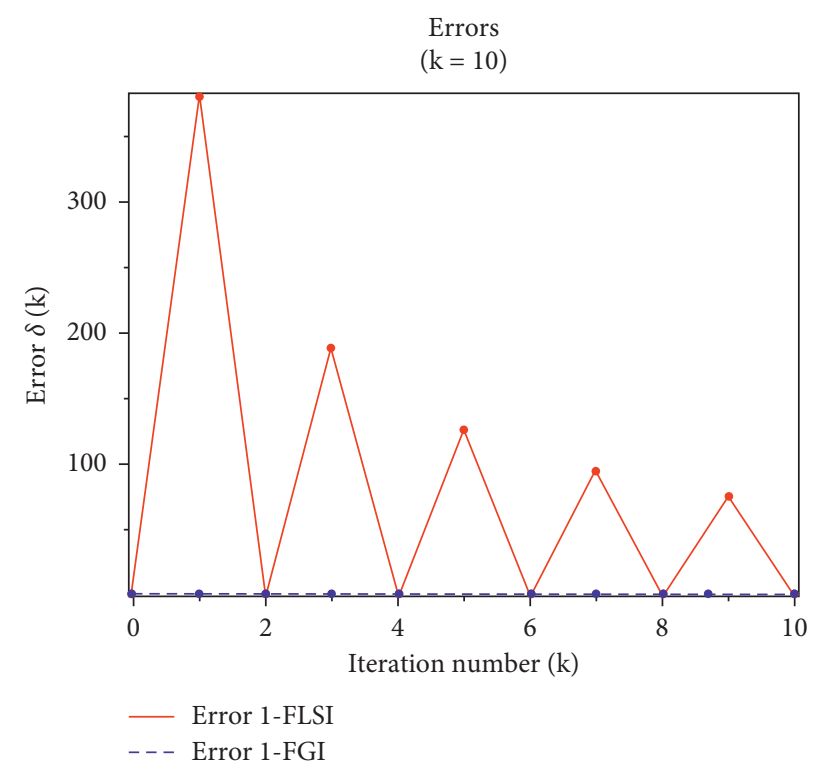

(a)

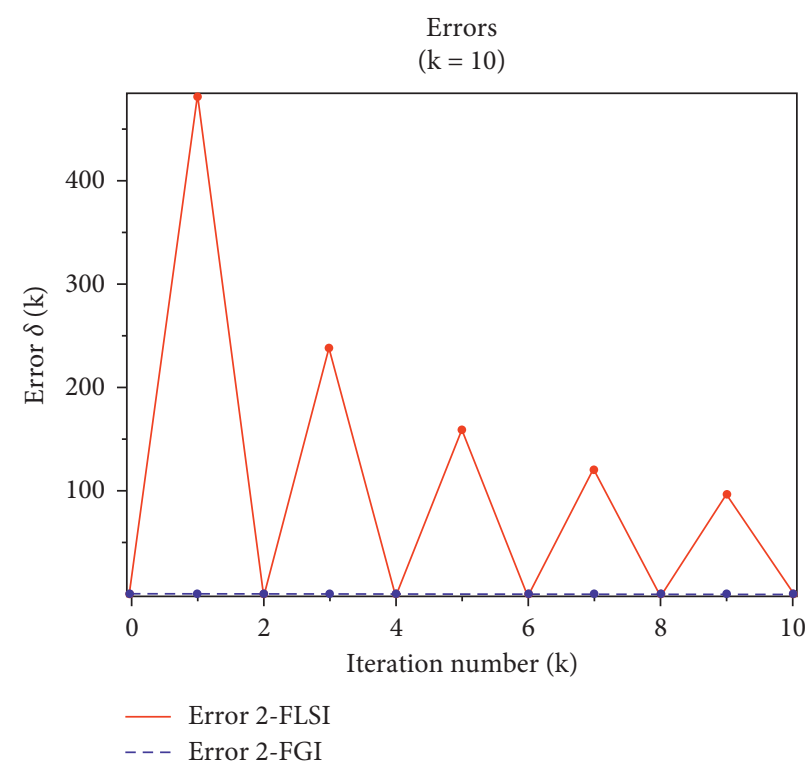

(b)

Figure 3: Continued. 


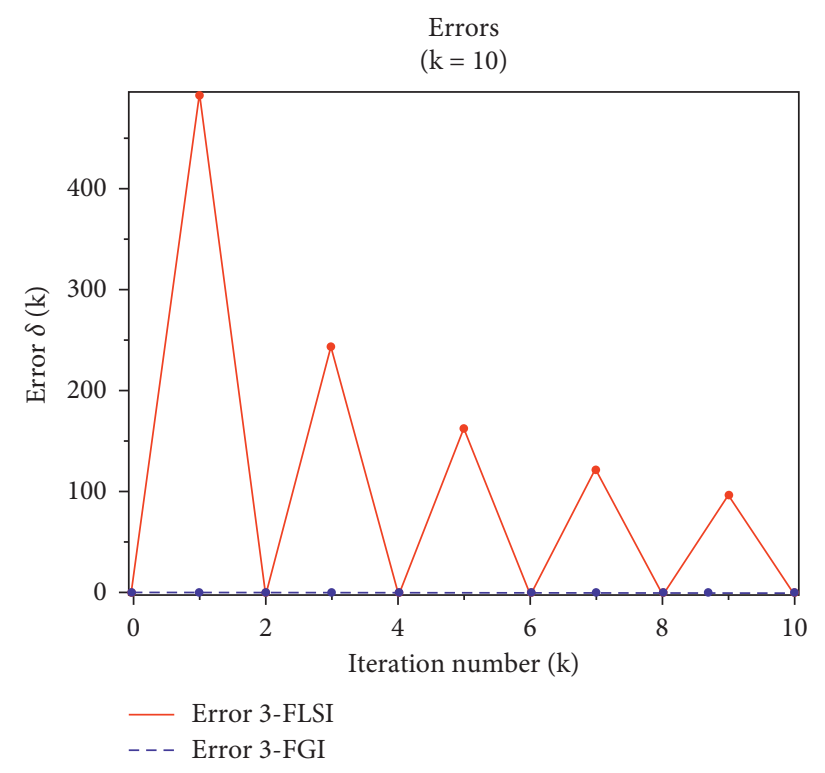

(c)

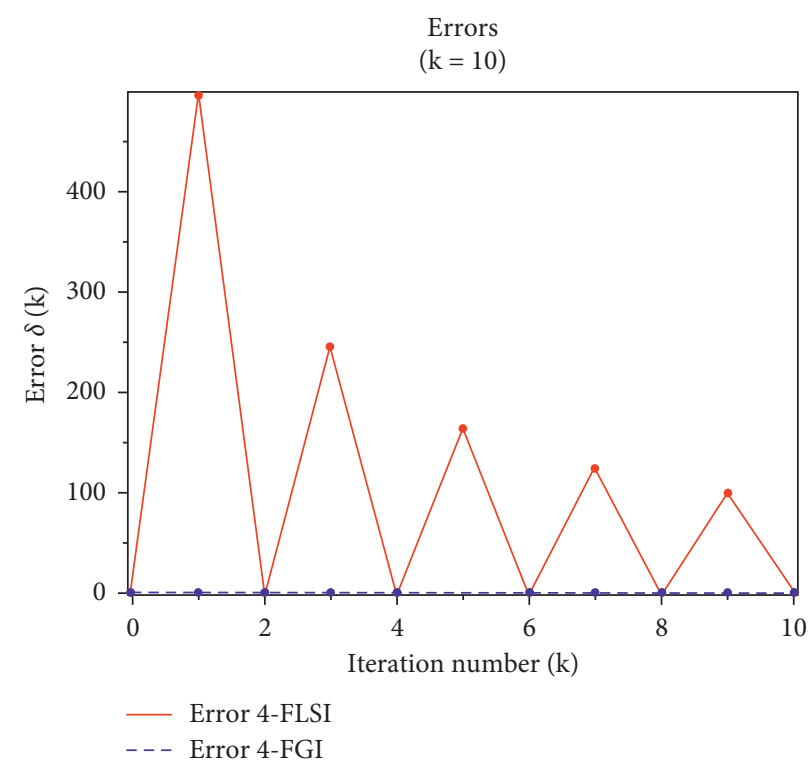

(d)

FIgURE 3: Comparison between relative $\delta^{l}(k)$ of FGI and FLSI for the first ten iterations for Example 2.

Solution. The solution for the given CTrFFSME is obtained by the proposed methods as follows.

4.4. Fuzzy Matrix Vectorization Method (FMVM). To apply FMVM, we need to find the inverse of the $10000 \times 10000$ matrix, which requires long computational timing and huge memory. Thus, FMVM is not a practical approach for such a large dimensional system.
4.5. Fuzzy Gradient Iterative (FGI) Method and Fuzzy LeastSquare Iterative (FLSI) Method. Algorithm 2 for FGI and FLSI is applied to compute the approximated fuzzy solution $\stackrel{\wedge}{X}^{(l)}(k)$ and $\hat{Y}^{(l)}(k)$ for the given CTrFFSME with $\alpha_{1}=\alpha_{2}=$ $\alpha_{3}=\alpha_{4}=0.999$ for FLSI and $\alpha_{1}=\alpha_{2}=\alpha_{3}=\alpha_{4}=2 \times 10^{-7}$ for FGI using the following initial value:

$$
\begin{aligned}
& \stackrel{\wedge}{X}^{(l)}(0)=\text { LinearAlgebra: }- \text { RandomMatrix }(100,100, \text { generator }=0) \\
& \stackrel{\wedge}{Y}^{(l)}(0)=\text { LinearAlgebra: }- \text { RandomMatrix }(100,100, \text { generator }=0)
\end{aligned}
$$

In Table 3, the computational time and memory usage for the first ten iterations for FLSI and FGI are compared.

Figure 3 shows the change in the relative error $\delta^{l}(k)$ when $k$ increases up to $k=10$.

From Table 3 and Figure 3, the relative error $\delta^{l}(k)$ is becoming smaller as $k$ increases. This indicates that the proposed methods are effective and convergent for the given CTrFFSME. This indicates that the proposed algorithms are effective and convergent for the given positive CTrFFSME.

\section{Conclusion}

Analyzing the stability of many control systems required solving a couple of crisp Sylvester matrix equations simultaneously. In this paper, we presented the solution to the CTrFFSME and its special cases analytically by FMVM and numerically by FGI and FLSI methods. The FMVM aims to find the exact solution. However, it is limited to small-sized CTrFFSME, while FGI and FLSI can find an approximated fuzzy solution to large CTrFFSME. The numerical example analysis and graphical representation of the relative error indicate that the approximated solutions obtained by FGI and FLSI algorithms converge to the exact solution for any initial value and any size of the matrix system (up to $100 \times 100$ ). In addition, the relative error is becoming smaller as the number of iterations increases. This indicates that the proposed methods are effective and convergent for the given CTrFFSME regardless of any size of matrices. In addition, the developed method can find positive fuzzy solutions to the following fuzzy equations:

(i) Couple fully fuzzy Sylvester matrix equations with positive trapezoidal and triangular fuzzy numbers

(ii) Couple fully fuzzy Lyapunov matrix equations with positive trapezoidal and triangular fuzzy numbers 
(iii) Couple fully fuzzy Sylvester matrix equations with positive trapezoidal and triangular fuzzy

(iv) Couple fully fuzzy Lyapunov matrix equations with positive trapezoidal and triangular fuzzy

(v) Couple fully fuzzy matrix equation with positive triangular and trapezoidal fuzzy numbers

The main disadvantage of this work is that it is limited to CTrFFSME with positive TrFNs only. As future research, the ideas presented in this paper will be extended to CTrFFSME with arbitrary trapezoidal fuzzy numbers.

\section{Data Availability}

No data were used to support this study.

\section{Additional Points}

This article does not contain any studies with human participants or animals performed by any of the authors.

\section{Consent}

Informed consent was obtained from all individual participants included in the study.

\section{Conflicts of Interest}

The authors declare that there are no conflicts of interest, financial, or otherwise.

\section{References}

[1] H. K. Wimmer, "Consistency of a pair of generalized sylvester equations," IEEE Transactions on Automatic Control, vol. 39, no. 5, pp. 1014-1016, 1994.

[2] A.-G. Wu, G.-R. Duan, and B. Zhou, "Solution to generalized Sylvester matrix equations," IEEE Transactions on Automatic Control, vol. 53, no. 3, pp. 811-815, 2008.

[3] L. Li Qin and T. Tongwen Chen, "Multirate sampled-data systems: all $\mathrm{H} /$ sub $\mathrm{O} /$ suboptimal controllers and the minimum entropy controller," IEEE Transactions on Automatic Control, vol. 44, no. 3, pp. 537-550, 1999.

[4] A. Saberi and P. Sannuti, Control of Linear Systems with Regulation and Input Constraints, Springer Science \& Business Media, Berlin, Heidelberg, Germany, 2000.

[5] M. Darouach, "Solution to Sylvester equation associated to linear descriptor systems," Systems and Control Letters, vol. 55, no. 10, pp. 835-838, 2006.

[6] S. Lesecq, A. Barraud, and N. Christov, "On the local sensitivity of the Lyapunov equations," in Lecture Notes in Computer ScienceSpringer, Berlin, Heidelberg, Germany, 2001.

[7] R.-C. Li, "A bound on the solution to a structured Sylvester equation with an application to relative perturbation theory," SIAM Journal on Matrix Analysis and Applications, vol. 21, no. 2, pp. 440-445, 2000.

[8] V. L. Syrmos and F. L. Lewis, "Coupled and constrained sylvester equations in system design," Circuits, Systems, and Signal Processing, vol. 13, no. 6, pp. 663-694, 1994.
[9] F. De Teran and F. Dopico, "The equation $X A+A X *=0$ and the dimension of $*$ congruence orbits," The Electronic Journal of Linear Algebra, vol. 22, pp. 448-465, 2011.

[10] B. Datta, Numerical Methods for Linear Control Systems, Academic Press, Cambridge, MA, USA, 2004.

[11] C. Paige and C. Van Loan, "A Schur decomposition for Hamiltonian matrices," Linear Algebra and its Applications, vol. 41, pp. 11-32, 1981.

[12] D. Calvetti and L. Reichel, "Application of ADI iterative methods to the restoration of noisy images," SIAM Journal on Matrix Analysis and Applications, vol. 17, no. 1, pp. 165-186, 1996.

[13] A. Bouhamidi and K. Jbilou, "Sylvester Tikhonov-regularization methods in image restoration," Journal of Computational and Applied Mathematics, vol. 206, no. 1, pp. 86-98, 2007.

[14] D. C. Sorensen and A. C. Antoulas, "The Sylvester equation and approximate balanced reduction," Linear Algebra and its Applications, vol. 351-352, pp. 671-700, 2002.

[15] S. Faizi, W. Sałabun, T. Rashid, J. Wątróbski, and S. Zafar, "Group decision-making for hesitant fuzzy sets based on characteristic objects method," Symmetry, vol. 9, no. 8, p. 136, 2017.

[16] T. Mori and H. Kokame, "On solution bounds for three types of Lyapunov matrix equations: continuous, discrete and unified equations," IEEE Transactions on Automatic Control, vol. 47, no. 10, pp. 1767-1770, 2002.

[17] P. Benner, "Control theory basics," 2007, https://labattmot. ele.ita.br/ele/alessandro/Leitura/04-PAPERS/ARTIGOS/ CONTROLEB\%C1SICO/ControlTheoryBasics.pdf.

[18] J. Heinen, "A technique for solving the extended discrete lyapunov matrix equation," IEEE Transactions on Automatic Control, vol. 17, no. 1, pp. 156-157, 1972.

[19] G. Golub, S. Nash, and C. van Loan, "A Hessenberg-Schur method for the problem $A X+X B=C$," IEEE Transactions on Automatic Control, vol. 24, no. 6, pp. 909-913, 1979.

[20] J.-J. Climent and C. Perea, "Convergence and comparison theorems for a generalized alternating iterative method," Applied Mathematics and Computation, vol. 143, no. 1, pp. 1-14, 2003.

[21] G. Starke and W. Niethammer, "SOR for $A X-X B=C$," Linear Algebra and its Applications, vol. 154-156, pp. 355-375, 1991.

[22] I. Jonsson and B. Kågström, "Recursive blocked algorithms for solving triangular systems-part II," ACM Transactions on Mathematical Software, vol. 28, no. 4, pp. 416-435, 2002.

[23] B. Kågström, "A perturbation analysis of the generalized sylvester equation $\$(A R-L B, D R-L E)=(C, F) \$$," SIAM Journal on Matrix Analysis and Applications, vol. 15, no. 4, pp. 1045-1060, 1994.

[24] F. Ding and T. Chen, "Iterative least-squares solutions of coupled Sylvester matrix equations," Systems and Control Letters, vol. 54, no. 2, pp. 95-107, 2005.

[25] Q.-W. Wang and Z.-H. He, "Systems of coupled generalized Sylvester matrix equations," Automatica, vol. 50, no. 11, pp. 2840-2844, 2014.

[26] S. K. Sheng-Kun Li, "An iterative method for the least squares symmetric solutions of the generalized coupled sylvester matrix equations," in Proceedings of the 2010 International Conference on Computer Application and System Modeling (ICCASM 2010), vol. 6, pp. 650-653, Taiyuan, China, October 2010.

[27] M. A. Ramadan and T. S. el-Danaf, "Solving the generalized coupled Sylvester matrix equations over generalized 
bisymmetric matrices," Transactions of the Institute of Measurement and Control, vol. 37, no. 3, pp. 291-316, 2015.

[28] F. Ding and T. Chen, "On iterative solutions of general coupled matrix equations," SIAM Journal on Control and Optimization, vol. 44, no. 6, pp. 2269-2284, 2006.

[29] M. Steeleworthy and P. T. Dewan, "Web-based citation management systems: which one is best?" Partnership: the Canadian Journal of Library and Information Practice and Research, vol. 8, no. 1, 2013.

[30] B. Huang and C. Ma, "An iterative algorithm for the least Frobenius norm least squares solution of a class of generalized coupled Sylvester-transpose linear matrix equations," Applied Mathematics and Computation, vol. 328, pp. 58-74, 2018.

[31] Y. Feng and M. Yagoubi, Robust Control of Linear Descriptor Systems, Springer, Berlin, Heidelberg, Germany, 2017, https:// www.springer.com/series/13304.

[32] Q.-W. Wang and Z.-H. He, "Solvability conditions and general solution for mixed Sylvester equations," Automatica, vol. 49, no. 9, pp. 2713-2719, 2013.

[33] X. Sheng, "A relaxed gradient based algorithm for solving generalized coupled Sylvester matrix equations," Journal of the Franklin Institute, vol. 355, no. 10, pp. 4282-4297, 2018.

[34] J. Hu and C. Ma, "Conjugate gradient least squares algorithm for solving the generalized coupled Sylvester-conjugate matrix equations," Applied Mathematics and Computation, vol. 334, pp. 174-191, 2018.

[35] L. Lv, Z. Zhang, L. Zhang, and X. Liu, "Gradient based approach for generalized discrete-time periodic coupled Sylvester matrix equations," Journal of the Franklin Institute, vol. 355, no. 15, pp. 7691-7705, 2018.

[36] T. Yan and C. Ma, "The BCR algorithms for solving the reflexive or anti-reflexive solutions of generalized coupled Sylvester matrix equations," Journal of the Franklin Institute, vol. 357, no. 17, pp. 12787-12807, 2020.

[37] M. Hajarian, "Finding solutions for periodic discrete-time generalized coupled Sylvester matrix equations via the generalized BCR method," Transactions of the Institute of Measurement and Control, vol. 40, no. 2, pp. 647-656, 2018.

[38] M. Hajarian, "Convergence properties of BCR method for generalized Sylvester matrix equation over generalized reflexive and anti-reflexive matrices," Linear and Multilinear Algebra, vol. 66, no. 10, pp. 1975-1990, 2018.

[39] M. Friedman, M. Ming, and A. Kandel, "Fuzzy linear systems," Fuzzy Sets and Systems, vol. 96, no. 2, pp. 201-209, 1998.

[40] T. Allahviranloo and S. Salahshour, "Fuzzy symmetric solutions of fuzzy linear systems," Journal of Computational and Applied Mathematics, vol. 235, no. 16, pp. 4545-4553, 2011.

[41] T. Allahviranloo, S. Salahshour, and M. Khezerloo, "Maximaland minimal symmetric solutions of fully fuzzy linear systems," Journal of Computational and Applied Mathematics, vol. 235, no. 16, pp. 4652-4662, 2011.

[42] G. Malkawi, N. Ahmad, H. Ibrahim, and D. J. Albayari, “A note on "solving fully fuzzy linear systems by using implicit Gauss-cholesky algorithm"," Computational Mathematics and Modeling, vol. 26, no. 4, pp. 585-592, 2015.

[43] G. Malkawi, N. Ahmad, and H. Ibrahim, "Solving fully fuzzy linear system with the necessary and sufficient condition to have a positive solution," Applied Mathematics and Information Sciences, vol. 8, no. 3, pp. 1003-1019, 2014.

[44] M. Otadi and M. Mosleh, "Solving fully fuzzy matrix equations," Applied Mathematical Modelling, vol. 36, no. 12, pp. 6114-6121, 2012.
[45] X. Guo and D. Shang, "Fuzzy approximate solution of positive fully fuzzy linear matrix equations," Journal of Applied Mathematics, vol. 2013, Article ID 178209, 7 pages, 2013.

[46] G. Malkawi, N. Ahmad, and H. Ibrahim, "Solving the fully fuzzy sylvester matrix equation with triangular fuzzy number," Far East Journal of Mathematical Sciences, vol. 98, no. 1, pp. 37-55, 2015.

[47] W. S. W. Daud, N. Ahmad, and G. Malkawi, "Positive fuzzy minimal solution for positive singular fully fuzzy Sylvester matrix equation," AIP Conference Proceedings, vol. 1974, Article ID 20084, 2018.

[48] A. A. A. Elsayed, N. Ahmad, and G. Malkawi, "On the solution of fully fuzzy Sylvester matrix equation with trapezoidal fuzzy numbers," Computational and Applied Mathematics, vol. 39, no. 4, pp. 1-22, 2020.

[49] A. Sadeghi, S. Abbasbandy, and M. E. Abbasnejad, "The common solution of the pair of fuzzy matrix equations," World Applied Sciences Journal, vol. 15, pp. 232-238, 2011.

[50] W. S. W. Daud, N. Ahmad, and G. Malkawi, "A preliminary study on the solution of fully fuzzy Sylvester matrix equation," AIP Conference Proceedings, vol. 1775, 2016.

[51] W. S. W. Daud, N. Ahmad, and G. Malkawi, "An algorithm for solving an arbitrary triangular fully fuzzy Sylvester matrix equations," AIP Conference Proceedings, vol. 1905, 2017.

[52] W. S. W. Daud, N. Ahmad, and G. Malkawi, "Positive solution of pair fully fuzzy matrix equations," Malaysian Journal of Mathematical Sciences, vol. 12, pp. 383-400, 2018.

[53] W. S. W. Daud, N. Ahmad, and G. Malkawi, "A new solution of pair matrix equations with arbitrary triangular fuzzy numbers," Turkish Journal of Mathematics, vol. 43, no. 3, pp. 1195-1217, 2019.

[54] L. Inearat and N. Qatanani, "Numerical methods for solving fuzzy linear systems," Mathematics, vol. 6, no. 2, p. 19, 2018.

[55] T. Allahviranloo, "Successive over relaxation iterative method for fuzzy system of linear equations," Applied Mathematics and Computation, vol. 162, no. 1, pp. 189-196, 2005.

[56] S. A. Edalatpanah, "Chebyshev semi-iterative method to solve fully fuzzy linear systems," Journal of Information and Computing Science, vol. 9, no. 1, pp. 67-74, 2014.

[57] S. Abbasbandy and A. Jafarian, "Steepest descent method for system of fuzzy linear equations," Applied Mathematics and Computation, vol. 175, no. 1, pp. 823-833, 2006.

[58] J. Gao and Q. Zhang, "A unified iterative scheme for solving fully fuzzy linear system," 2009 WRI Global Congress on Intelligent Systems, vol. 1, pp. 431-435, 2009.

[59] A. Syaiful Abidin, M. Mashadi, and S. Gemawati, "Algebraic modification of trapezoidal fuzzy numbers to complete fully fuzzy linear equations system using Gauss-Jacobi method," International Journal of Management and Fuzzy Systems, vol. 5, no. 2, p. 40, 2019.

[60] J.-F. Yin and K. Wang, "Splitting iterative methods for fuzzy system of linear equations," Computational Mathematics and Modeling, vol. 20, no. 3, pp. 326-335, 2009.

[61] M. Dehghan and B. Hashemi, "Iterative solution of fuzzy linear systems," Applied Mathematics and Computation, vol. 175, no. 1, pp. 645-674, 2006.

[62] S. A. Edalatpanah, "Modified iterative methods for solving fully fuzzy linear systems," Fuzzy Systems, vol. 1-3, pp. 55-73, 2017.

[63] T. Allahviranloo, "Numerical methods for fuzzy system of linear equations," Applied Mathematics and Computation, vol. 155, no. 2, pp. 493-502, 2004. 
[64] D. Dubois and H. Prade, "Operations on fuzzy numbers," International Journal of Systems Science, vol. 9, no. 6, pp. 613-626, 1978.

[65] A. Kaufmann and M. Gupta, Introduction to Fuzzy Arithmetic, Van Nostrand Reinhold Company, New York, NY, USA, 1991.

[66] K. H. Lee, "First course on fuzzy theory and applications," First Course on Fuzzy Theory and Applications Choice Reviews Online, Springer Science \& Business Media, vol. 42, no. 10, , pp. 42-5917, Berlin, Heidelberg, Germany, 2005.

[67] A. R. Meenakshi, Fuzzy Matrix: Theory and Applications, MJP Publisher, Chennai, India, 1st edition, 2019.

[68] H. Minc, Nonnegative Matrices, Wiley, Hoboken, NJ, USA, 1988.

[69] T. Otsu, "Matrix Algebra," in Econometric Theory, K. M. Abadir and J. R. Magnus, Eds., Cambridge University Press, Cambridge, UK, 2005.

[70] F. Ding, P. X. Liu, and J. Ding, "Iterative solutions of the generalized Sylvester matrix equations by using the hierarchical identification principle," Applied Mathematics and Computation, vol. 197, no. 1, pp. 41-50, 2008.

[71] M. A. Ramadan, T. S. El-Danaf, and A. M. E. Bayoumi, "Two iterative algorithms for the reflexive and Hermitian reflexive solutions of the generalized Sylvester matrix equation," Journal of Vibration and Control, vol. 21, no. 3, pp. 483-492, 2015.

[72] G. Malkawi, N. Ahmad, and H. Ibrahim, "Finite solutions of fully fuzzy linear system," AIP Conference Proceedings, vol. 1635, pp. 447-454, 2014. 